\title{
Quantum instantons with classical moduli spaces
}

\author{
Igor Frenkel \\ Yale University \\ Department of Mathematics \\ 10 Hillhouse Avenue \\ New Haven, CT 06520-8283 USA \\ Marcos Jardim \\ University of Massachusetts at Amherst \\ Department of Mathematics and Statistics \\ Amherst, MA 01003-9305 USA
}

October 28, 2018

\begin{abstract}
We introduce a quantum Minkowski space-time based on the quantum group $S U(2)_{q}$ extended by a degree operator and formulate a quantum version of the anti-self-dual Yang-Mills equation. We construct solutions of the quantum equations using the classical ADHM linear data, and conjecture that, up to gauge transformations, our construction yields all the solutions. We also find a deformation of Penrose's twistor diagram, giving a correspondence between the quantum Minkowski space-time and the classical projective space $\mathbb{P}^{3}$.
\end{abstract}




\section{Introduction}

Our present view of the mathematical structure of space-time was first formulated by H. Minkowski in [21, based on Einstein's discovery of Special Relativity. Since then, many mathematicians and physicists have tried to develop various generalizations of Minkowski's concept of space-time, in different directions.

One such generalization was strongly advocated by R. Penrose [28]. He studied the conformal compactification of the complexified Minkowski spacetime, denoted by $\mathbb{M}$, and the associated space of null straight lines, denoted by $\mathbb{P}$, showing that various equations from Mathematical Physics (e.g. Maxwell and Dirac equations) over $\mathbb{M}$ can be transformed into natural holomorphic objects over $\mathbb{P}$.

One of the most celebrated examples of the Penrose programme was the solution of the anti-self-dual Yang-Mills (ASDYM) equations by M. Atiyah, V. Drinfeld, N. Hitchin and Yu. Manin. These authors described explicitly the moduli space $\mathcal{M}^{\text {reg }}(n, c)$ of finite action solutions of the ASDYM equation over $\mathbb{R}^{4}$, usually called instantons, of fixed rank $n$ and charge $c$ in terms of some linear data [1]. In particular, they constructed an instanton associated to any linear data, and proved, conversely, that every instanton can be obtained in this way, up to gauge transformations.

The Penrose approach has also been successfully applied to massless linear equations, the full Yang-Mills equation, self-dual Einstein equation, etc. (see 31] and the references therein).

Another generalization of the Minkowski space-time based on noncommutative geometry was proposed by A. Connes [5]. The first step in this approach is the replacement of geometric objects by their algebras of functions and the reformulation of various geometric concepts in algebraic language. The next step is the deformation of the algebraic structures and the introduction of noncommutativity. The algebraic structures so obtained are no longer associated with the original geometry, being regarded as geometric 
structures on a "noncommutative space".

The simplest example of noncommutative space-time can be obtained by replacing the commutative algebra $\mathbb{R}^{4}$ by the Heisenberg algebra. Recently, N. Nekrasov and A. Schwarz [26] have defined instantons on such noncommutative $\mathbb{R}^{4}$ and argued that the corresponding moduli space can be parametrized by a modification of the Atiyah, Drinfeld, Hitchin and Manin (ADHM) linear data.

Another source for the deformation of the Minkowski space-time emerged with the discovery of quantum groups by V. Drinfeld [10] and M. Jimbo [13. It quickly led to the notion of a quantum Minkowski space-time and various related structures; some of the early papers are [3, 18, 29]. In particular, a quantum version of the ASDYM equation was studied in 35.

One expects that the linear data of Atiyah, Drinfeld, Hitchin and Manin should also be deformed in order to yield solutions of the quantum ASDYM equation. Thus the quantum Minkowski space-time and related structures, though mathematically sensible, seem rather dubious in terms of physical applications. In fact, the quantum deformation destroys the classical symmetry groups and the possible reconstruction of these symmetries is far from apparent.

In the present paper we observe a new phenomenon, which goes against the typical intuition related to quantum deformations of the Minkowski space-time and various equations on it. We show that there exists a natural quantum deformation of the Minkowski space-time (in fact, of the whole compactified complexified Minkowski space $\mathbb{M}$, along with its real structures) and the ASDYM equation, such that the moduli space of quantum instantons is naturally parameterized by the classical, non-deformed ADHM data.

Starting from the classical ADHM data, we will explicitly construct solutions of the quantum ASDYM equation. Furthermore, we conjecture that our construction yields all the solutions up to gauge equivalence. We hope to prove this conjecture using a generalization of the Penrose twistor trans- 
form, which takes the quantum Minkowski space-time to the classical space of straight lines. Such procedure thus realizes Penrose's dream, who regarded light rays as more fundamental than points in space-time: in our construction, space-time is being deformed while the space of light rays is kept fixed.

Moreover, this phenomenon of quantum equations with classical solutions does not seem to be restricted only to the ASDYM equation, but it is also steadfast for massless linear equations, full Yang-Mills equations, etc. This opens new venues for physical applications of the quantum Minkowski spacetime here proposed: the classical symmetry groups can be restored if one considers all quantum deformations of the classical Minkowski space-time, since the spaces of solutions of the quantum equations admit natural identifications.

Our constructions are based on the theory of the quantum group $S U(2)_{q}$ extended by a natural degree operator, which makes possible for a surprising construction of solutions of the quantum ASDYM equation from the classical ADHM data. The extended quantum group $\widetilde{S U}(2)_{q}$ has functional dimension 4 , the same as the quantum space-time, and we show that the relation between them extends to deep structural levels.

To formulate the ASDYM equation we need a theory of exterior forms on quantum Minkowski space-time. This is derived from the differential calculus on $S U(2)_{q}$ first developed in [32, 34, with the addition of the differential of the degree operator. The $R$-matrix formulation of the quantum group $S U(2)_{q}$ [11] and its exterior algebra [30] allows us to present the construction of the quantum connections even more compactly than in the classical case, and efficiently verify the quantum ASDYM equation.

Our results bring us to the conclusion that the correct notion for a quantum Minkowski space-time is precisely the extended quantum group $\widetilde{S U}(2)_{q}$. This relation, which might seem artificial at the classical level $(q=1)$, is imposed on us by the mathematical structure itself. We believe that future research will reveal the full potential of this new incarnation of the Minkowski 
space-time.

\section{Contents}

1 Quantum Minkowski space-time from $S U(2)$

1.1 Algebraic structures on Minkowski space-time . . . . . . . . . 5

1.2 The quantum group $S U(2)$ and its extension . . . . . . . . . 8

1.3 Quantum Minkowski space-time . . . . . . . . . . . . . . . . 12

2 Differential forms on quantum Minkowski space-time $\quad 15$

2.1 Differential forms on Minkowski space-time. . . . . . . . . 15

2.2 Differential forms on the quantum group $S U(2) \ldots$. . . . . . . 17

2.3 Differential forms on quantum Minkowski space-time . . . . . 20

3 Construction of quantum instantons 23

3.1 Classical instantons and the ADHM data . . . . . . . . . . . 23

3.2 Quantum Haar measure and duality . . . . . . . . . . . . . 26

3.3 Construction of quantum instantons . . . . . . . . . . . . . . . 28

4 Quantum Penrose transform and further perspectives 38

4.1 Completeness coniecture and the guantum Penrose transform 38

4.2 Remarks on roots of unity and representation theory . . . . . 49

4.3 Further perspectives . . . . . . . . . . . . . . . . . 51

References $\quad 52$

\section{Quantum Minkowski space-time from $S U(2)_{q}$}

\subsection{Algebraic structures on Minkowski space-time}

We begin with some well known facts regarding Penrose's approach to the Minkowski space-time for the convenience of the reader; further details can be found in [19, 31]. 
Let $\mathbb{T}$ be a 4 dimensional complex vector space and consider $\mathbb{M}=\mathbf{G}_{2}(\mathbb{T})$, the Grassmannian of planes in $\mathbb{T}$. As usual in the literature, we will often refer to $\mathbb{M}$ as the compactified complexified Minkowski space, since $\mathbb{M}$ can be obtained via a conformal compactification of $M^{4} \otimes \mathbb{C}$, where $M^{4}$ denotes the usual Minkowski space.

The Grassmannian $\mathbb{M}$ can be realized as a quadric in $\mathbb{P}^{5}$ via the Plücker embedding. More precisely, note that $\mathbf{P}\left(\Lambda^{2} \mathbb{T}\right) \simeq \mathbb{P}^{5}$, and take homogeneous coordinates $\left[z_{r s}\right]$ for $r, s=1,2,3,4$ (where $z_{r s}=-z_{s r}$ is the coefficient of $\left.d z_{r} \wedge d z_{s}\right)$. Then $\mathbb{M}$ becomes the subvariety of $\mathbf{P}\left(\Lambda^{2} \mathbb{T}\right)$ given by the quadric:

$$
z_{12} z_{34}-z_{13} z_{24}+z_{14} z_{23}=0
$$

Let us now fix a direct sum decomposition of $\mathbb{T}$ into two 2-dimensional subspaces:

$$
\mathbb{T}=\mathbb{L} \oplus \mathbb{L}^{\prime}
$$

Such choice induces a decomposition of the second exterior power as follows:

$$
\Lambda^{2} \mathbb{T}=\Lambda^{2} \mathbb{L} \oplus \Lambda^{2} \mathbb{L}^{\prime} \oplus \mathbb{L} \wedge \mathbb{L}^{\prime}
$$

Now fix basis $\left\{e_{1}, e_{2}\right\}$ and $\left\{e_{1^{\prime}}, e_{2^{\prime}}\right\}$ in $\mathbb{L}$ and $\mathbb{L}^{\prime}$, respectively. To help us keep track of the choices made, we will use indexes $\left\{1,2,1^{\prime}, 2^{\prime}\right\}$ instead of $\{1,2,3,4\}$. Since the variables $z_{12}$ and $z_{1^{\prime} 2^{\prime}}$ will play a very special role in our discussion, we will introduce the notation $D=z_{12}$ and $D^{\prime}=z_{34}=z_{1^{\prime} 2^{\prime}}$. The quadric (1I) is then rewritten in the following way:

$$
z_{11^{\prime}} z_{22^{\prime}}-z_{12^{\prime}} z_{21^{\prime}}=D D^{\prime}
$$

The decomposition (21) also induces the choice of a point at infinity in the compactified complexified Minkowski space $\mathbb{M}$. Let $\mathcal{S}(x)$ denote the plane in $\mathbb{T}$ corresponding to the point $x \in \mathbb{M}$ and $\ell$ denote the point in $\mathbb{M}$ corresponding to the plane $\mathbb{L}$. Consider the sets:

$$
\begin{aligned}
\mathbb{M}^{\mathrm{I}}=\{x \in \mathbb{M} \mid \mathcal{S}(x) \cap \mathbb{L}=\{0\}\} & \text { complexified Minkowski space } \\
C(\ell)=\{x \in \mathbb{M} \mid \operatorname{dim}(\mathcal{S}(x) \cap \mathbb{L})=1\} & \text { light cone at infinity }
\end{aligned}
$$


Then clearly $\mathbb{M}=\mathbb{M}^{\mathrm{I}} \cup C(\ell) \cup\{\ell\}$, and $\mathbb{M}^{\mathrm{I}}$ is an affine space, being isomorphic to $\mathbb{C}^{4}=M^{4} \otimes \mathbb{C}$. Moreover, we note that the light cone at infinity $C(\ell)$ has complex codimension one in $\mathbb{M}$.

We will denote the local coordinates on $\mathbb{M}^{\mathrm{I}}$ by $x_{r s^{\prime}}=z_{r s^{\prime}} / D$, where $r, s=1,2$. They are related to the Euclidean coordinates $x^{k}$ on $\mathbb{M}^{\mathrm{I}}$ in the following way:

$$
\begin{gathered}
x_{11^{\prime}}=x^{1}-i x^{4} \quad x_{12^{\prime}}=-i x^{2}-x^{3} \\
x_{21^{\prime}}=-i x^{2}+x^{3} \\
x_{22^{\prime}}=x^{1}+i x^{4}
\end{gathered}
$$

We will denote by $E^{4}$ the real Euclidean space spanned by $x^{k}$.

Similarly, let $\ell^{\prime}$ be the point in $\mathbb{M}$ corresponding to the plane $\mathbb{L}^{\prime}$. It can be regarded as the origin in $\mathbb{C}^{4}$. We define:

$$
\begin{gathered}
\mathbb{M}^{\mathrm{J}}=\left\{x \in \mathbb{M} \mid \mathcal{S}(x) \cap \mathbb{L}^{\prime}=\{0\}\right\} \\
C\left(\ell^{\prime}\right)=\left\{x \in \mathbb{M} \mid \operatorname{dim}\left(\mathcal{S}(x) \cap \mathbb{L}^{\prime}\right)=1\right\}
\end{gathered}
$$

so that $\mathbb{M}^{\mathrm{J}}$ is also a 4-dimensional affine space and $\mathbb{M}=\mathbb{M}^{\mathrm{J}} \cup C\left(\ell^{\prime}\right) \cup\left\{\ell^{\prime}\right\}$. We will denote the local coordinates on $\mathbb{M}^{\mathrm{J}}$ by $y_{r s^{\prime}}=z_{r s^{\prime}} / D^{\prime}$, where $r, s=1,2$.

It is important to note that even though the affine spaces $\mathbb{M}^{\mathrm{I}}$ and $\mathbb{M}^{\mathrm{J}}$ do not cover the entire compactified complexified Minkowski space $\mathbb{M}$, only a codimension two submanifold is left out, since $\mathbb{M} \backslash\left(\mathbb{M}^{\mathbb{I}} \cup \mathbb{M}^{J}\right)=C(\ell) \cap C\left(\ell^{\prime}\right)$.

The intersection $\mathbb{M}^{\mathrm{IJ}}=\mathbb{M}^{\mathrm{I}} \cap \mathbb{M}^{\mathrm{J}}$ is given by the set of all $x \in \mathbb{M}^{\mathrm{I}}$ such that $\operatorname{det}(X)=x_{11^{\prime}} x_{22^{\prime}}-x_{12^{\prime}} x_{21^{\prime}} \neq 0$. Equivalently, this is also the set of all $y \in \mathbb{M}^{\mathrm{J}}$ such that $\operatorname{det}(Y)=y_{11^{\prime}} y_{22^{\prime}}-y_{12^{\prime}} y_{21^{\prime}} \neq 0$. The gluing map $\tau: \mathbb{M}^{\mathrm{IJ}} \rightarrow \mathbb{M}^{\mathrm{IJ}}$ relates the local coordinates on $\mathbb{M}^{\mathrm{I}}$ and $\mathbb{M}^{\mathrm{J}}$ in the following way:

$$
\begin{array}{rlrl}
x_{11^{\prime}} & =\frac{y_{22^{\prime}}}{\operatorname{det}(Y)} & x_{12^{\prime}} & =-\frac{y_{12^{\prime}}}{\operatorname{det}(Y)} \\
x_{21^{\prime}}=-\frac{y_{21^{\prime}}}{\operatorname{det}(Y)} & x_{22^{\prime}} & =\frac{y_{11^{\prime}}}{\operatorname{det}(Y)}
\end{array}
$$

Since $\mathbb{M}$ is an algebraic variety, it can also be characterized via its homogeneous coordinate algebra:

$$
\mathfrak{M}=\mathbb{C}\left[D, D^{\prime}, z_{11^{\prime}}, z_{12^{\prime}}, z_{21^{\prime}}, z_{22^{\prime}}\right]_{h} / \mathcal{I}_{m}
$$


where $\mathcal{I}_{m}$ is the ideal generated by the quadric (3). The subscript " $h$ " means that $\mathfrak{M}$ consists only of the homogeneous polynomials.

In this picture, the coordinate algebras of the affine varieties $\mathbb{M}^{\mathrm{I}}$ and $\mathbb{M}^{\mathrm{J}}$ introduced above can be interpreted as certain localizations of the quadratic algebra $\mathfrak{M}$. Indeed, the coordinate rings for $\mathbb{M}^{\mathrm{I}}$ and $\mathbb{M}^{\mathrm{J}}$ are respectively given by:

$$
\begin{aligned}
& \mathfrak{M}^{\mathrm{I}}=\mathfrak{M}\left[D^{-1}\right]_{0}=\mathbb{C}\left[x_{11^{\prime}}, x_{12^{\prime}}, x_{21^{\prime}}, x_{22^{\prime}}\right] \\
& \mathfrak{M}^{\mathrm{J}}=\mathfrak{M}\left[D^{\prime-1}\right]_{0}=\mathbb{C}\left[y_{11^{\prime}}, y_{12^{\prime}}, y_{21^{\prime}}, y_{22^{\prime}}\right]
\end{aligned}
$$

where the subscript "0" means that we take only the degree zero part of the localized graded algebra.

Finally, we note that $\mathfrak{M}^{\mathrm{I}}$ and $\mathfrak{M}^{\mathrm{J}}$ can be made isomorphic by adjoining the inverses of the determinants $\operatorname{det}(X)$ and $\operatorname{det}(Y)$, respectively. Indeed, define the matrices of generators:

$$
X=\left(\begin{array}{ll}
x_{11^{\prime}} & x_{12^{\prime}} \\
x_{21^{\prime}} & x_{22^{\prime}}
\end{array}\right) \quad Y=\left(\begin{array}{cc}
y_{22^{\prime}} & -y_{12^{\prime}} \\
-y_{21^{\prime}} & y_{11^{\prime}}
\end{array}\right)
$$

The map $\eta: \mathfrak{M}^{\mathrm{I}}\left[\operatorname{det}(X)^{-1}\right] \rightarrow \mathfrak{M}^{\mathrm{J}}\left[\operatorname{det}(Y)^{-1}\right]$ given by:

$$
\eta(X)=\frac{Y}{\operatorname{det}(Y)}
$$

is an isomorphism. It is the algebraic analogue of the gluing map $\tau$ described above in geometric context, while $\mathfrak{M}^{\mathrm{I}}\left[\operatorname{det}(X)^{-1}\right] \simeq \mathfrak{M}^{\mathrm{J}}\left[\operatorname{det}(Y)^{-1}\right]$ plays the role of the intersection $\mathbb{M}^{\mathrm{IJ}}=\mathbb{M}^{\mathrm{I}} \cap \mathbb{M}^{\mathrm{J}}$.

\subsection{The quantum group $S U(2)_{q}$ and its extension}

Let $q$ be a formal parameter. Recall that the quantum group $G L(2)_{q}$ is the bialgebra over $\mathbb{C}$ generated by $g_{11^{\prime}}, g_{12^{\prime}}, g_{21^{\prime}}, g_{22^{\prime}}$ subject to the following commutation relations, see e.g. [4]:

$$
\begin{array}{ll}
g_{11^{\prime}} g_{12^{\prime}}=q^{-1} g_{12^{\prime}} g_{11^{\prime}} & g_{11^{\prime}} g_{21^{\prime}}=q^{-1} g_{21^{\prime}} g_{11^{\prime}} \\
g_{12^{\prime}} g_{22^{\prime}}=q^{-1} g_{22^{\prime}} g_{12^{\prime}} & g_{21^{\prime}} g_{22^{\prime}}=q^{-1} g_{22^{\prime}} g_{21^{\prime}}
\end{array}
$$




$$
\begin{gathered}
g_{12^{\prime}} g_{21^{\prime}}=g_{21^{\prime}} g_{12^{\prime}} \\
g_{11^{\prime}} g_{22^{\prime}}-q^{-1} g_{12^{\prime}} g_{21^{\prime}}=g_{22^{\prime}} g_{11^{\prime}}-q g_{21^{\prime}} g_{12^{\prime}}
\end{gathered}
$$

The comultiplication $\Delta$ and the counit $\varepsilon$ are given by:

$$
\begin{gathered}
\Delta\left(g_{r s^{\prime}}\right)=\sum_{k=1,2} g_{r k^{\prime}} \otimes g_{k s^{\prime}}, \quad r, s=1,2 \\
\varepsilon\left(g_{r s^{\prime}}\right)=\delta_{r s^{\prime}}, \quad r, s=1,2
\end{gathered}
$$

The expression (17) is called the quantum determinant and it is denoted by $\operatorname{det}_{q}(g)$. One easily checks that:

$$
\begin{gathered}
g_{r s^{\prime}} \operatorname{det}_{q}(g)=\operatorname{det}_{q}(g) g_{r s^{\prime}}, \quad r, s=1,2 \\
\Delta\left(\operatorname{det}_{q}(g)\right)=\operatorname{det}_{q}(g) \otimes \operatorname{det}_{q}(g)
\end{gathered}
$$

In order to define a Hopf algebra structure on $G L(2)_{q}$ we adjoin the inverse of the quantum determinant $\operatorname{det}_{q}(g)^{-1}$ to the generators $g_{r s^{\prime}}$ with the obvious relations. Then the antipode exists, and it is given by:

$$
\begin{array}{cc}
\gamma\left(g_{11^{\prime}}\right)=g_{22^{\prime}} \operatorname{det}_{q}(g)^{-1} & \gamma\left(g_{12^{\prime}}\right)=-q g_{12^{\prime}} \operatorname{det}_{q}(g)^{-1} \\
\gamma\left(g_{21^{\prime}}\right)=-q^{-1} g_{21^{\prime}} \operatorname{det}_{q}(g)^{-1} & \gamma\left(g_{22^{\prime}}\right)=g_{11^{\prime}} \operatorname{det}_{q}(g)^{-1}
\end{array}
$$

We will assume that the quantum group $G L(2)_{q}$ contains $\operatorname{det}_{q}(g)^{-1}$ and is therefore a Hopf algebra.

The quantum group $S L(2)_{q}$ is defined by the quotient:

$$
S L(2)_{q}=G L(2)_{q} /\left\langle\operatorname{det}_{q}(g)=1\right\rangle
$$

It is a well defined Hopf algebra by equations (10) and (11).

It is also useful to re-express the commutation relations for the quantum group $G L(2)_{q}$ by means of the $R$-matrix; let

$$
R_{12}=\left(\begin{array}{cccc}
p^{-1} & 0 & 0 & 0 \\
0 & 1 & p^{-1}-q & 0 \\
0 & p^{-1}-q^{-1} & 1 & 0 \\
0 & 0 & 0 & p^{-1}
\end{array}\right), \quad p=q^{ \pm 1}
$$


and let $T$ be the matrix of generators, i.e.:

$$
T=\left(\begin{array}{ll}
g_{11^{\prime}} & g_{12^{\prime}} \\
g_{21^{\prime}} & g_{22^{\prime}}
\end{array}\right)
$$

Then the relations (6) and (7) can then be put in the following compact form:

$$
R_{12} T_{1} T_{2}=T_{2} T_{1} R_{12}
$$

where $T_{1}=T \otimes 1$ and $T_{2}=\mathbf{1} \otimes T$ [11. Note that the commutation relations (66) and (77) do not depend on the parameter $p$. The $R$-matrix (12) also satisfies the Hecke relation:

$$
R_{12}-\left(R_{21}\right)^{-1}=\left(p^{-1}-p\right) P
$$

where $R_{21}=R_{12}^{\mathrm{t}}$ (with the superscript "t" meaning transposition) and $P$ is the permutation matrix. Equivalently, we also have for $\hat{R}_{12}=P R_{12}$ :

$$
\left(\hat{R}_{12}\right)^{2}=\left(p^{-1}-p\right) \hat{R}_{12}+\mathbf{1}
$$

Next, we will extend the quantum groups $G L(2)_{q}$ and $S L(2)_{q}$ by introducing a new generator $\delta$ and its inverse, satisfying the following commutation relations with the quantum group generators:

$$
\begin{array}{cc}
\delta g_{11^{\prime}}=g_{11^{\prime}} \delta & \delta g_{12^{\prime}}=q g_{12^{\prime}} \delta \\
\delta g_{21^{\prime}}=q^{-1} g_{21^{\prime}} \delta & \delta g_{22^{\prime}}=g_{22^{\prime}} \delta
\end{array}
$$

In matrix form, the above relations become:

$$
\delta T Q^{2}=Q^{2} T \delta
$$

where $Q$ is the following matrix:

$$
Q=\left(\begin{array}{cc}
q^{\frac{1}{4}} & 0 \\
0 & q^{-\frac{1}{4}}
\end{array}\right)
$$

Strictly speaking, one should consider $q^{\frac{1}{4}}$ to be the formal parameter, instead of $q$. However, fractional powers of $q$ will rarely appear in this paper. 
In other words, we define

$$
\widetilde{G L}(2)_{q}=G L(2)_{q}\left[\delta, \delta^{-1}\right] /(15) \text { and } \widetilde{S L}(2)_{q}=S L(2)_{q}\left[\delta, \delta^{-1}\right] /(15)
$$

The comultiplication, counit and antipode in $\widetilde{G L}(2)_{q}$ and $\widetilde{S L}(2)_{q}$ are given by:

$$
\Delta(\delta)=\delta \otimes \delta, \quad \varepsilon(\delta)=1, \quad \gamma(\delta)=\delta^{-1}
$$

It is easy to check that $\widetilde{G L}(2)_{q}$ and $\widetilde{S L}(2)_{q}$ satisfy the axioms of a Hopf algebra, and can thus be thought as quantum groups. Besides, we have the identity:

$$
\gamma^{2}(g)=\delta^{2} g \delta^{-2}, \quad \text { for all } g \in G L(2)_{q}, S L(2)_{q}
$$

Thus conjugation by $\delta$ can be viewed as a square root of the antipode squared.

In this paper, we will be primarily interested in the quantum group $S L(2)_{q}$ and its extension $\widetilde{S L}(2)_{q}$. For these quantum groups we define an involution $\dagger$ which fixes the formal parameter (i.e. $q^{\dagger}=q$ ) and acts on the generators as follows:

$$
\begin{array}{cc}
g_{11^{\prime}}^{\dagger}=g_{22^{\prime}}, & g_{12^{\prime}}^{\dagger}=-g_{21^{\prime}} \\
g_{21^{\prime}}^{\dagger}=-g_{12^{\prime}}, & g_{22^{\prime}}^{\dagger}=g_{11^{\prime}} \\
\delta^{\dagger}=\delta, & \left(\delta^{-1}\right)^{\dagger}=\delta^{-1}
\end{array}
$$

We extend the f-involution to the quantum group $\widetilde{S L}(2)_{q}$ by requiring it to be a conjugate linear anti-homomorphism, that is:

$$
\begin{aligned}
& (x y)^{\dagger}=y^{\dagger} x^{\dagger}, \text { where } x, y \in \widetilde{S L}(2)_{q} ; \\
& a^{\dagger}=\bar{a}, \quad \text { for all } a \in \mathbb{C} .
\end{aligned}
$$

We define $S U(2)_{q}$ and $\widetilde{S U}(2)_{q}$ as the quantum groups $S L(2)_{q}$ and $\widetilde{S L}(2)_{q}$, respectively, equipped with the $\dagger$-involution:

$$
S U(2)_{q}=\left(S L(2)_{q}, \dagger\right) \text { and } \widetilde{S U}(2)_{q}=\left(\widetilde{S L}(2)_{q}, \dagger\right)
$$

Note that instead of the formal parameter $q$ in the definitions of the quantum groups, we could have used a positive real number, which is automaticaly fixed by the involution $\dagger$. However, we prefer to consider various specializations of formal $q$ later on, the most interesting one being the specialization to a root of unity (see also Section 4.2 below). 


\subsection{Quantum Minkowski space-time}

Let us introduce two new sets of variables on the extended quantum group $\widetilde{S L}(2)_{q}$ :

$$
\begin{array}{lr}
x_{11^{\prime}}=\delta g_{11^{\prime}}=g_{11^{\prime}} \delta, & x_{12^{\prime}}=q^{-1 / 2} \delta g_{12^{\prime}}=q^{1 / 2} g_{12^{\prime}} \delta, \\
x_{21^{\prime}}=q^{1 / 2} \delta g_{21^{\prime}}=q^{-1 / 2} g_{21^{\prime}} \delta, & x_{22^{\prime}}=\delta g_{22^{\prime}}=g_{22^{\prime}} \delta
\end{array}
$$

and

$$
\begin{array}{lrl}
y_{11^{\prime}}=\delta^{-1} g_{11^{\prime}}=g_{11^{\prime}} \delta^{-1}, & y_{12^{\prime}}=q^{1 / 2} \delta^{-1} g_{12^{\prime}}=q^{-1 / 2} g_{12^{\prime}} \delta^{-1}, \\
y_{21^{\prime}}=q^{-1 / 2} \delta^{-1} g_{21^{\prime}}=q^{1 / 2} g_{21^{\prime}} \delta^{-1}, & y_{22^{\prime}}=\delta^{-1} g_{22^{\prime}}=g_{22^{\prime}} \delta^{-1}
\end{array}
$$

It is easy to determine their commutation relations:

$$
\begin{gathered}
x_{11^{\prime}} x_{12^{\prime}}=x_{12^{\prime}} x_{11^{\prime}}, \quad x_{21^{\prime}} x_{22^{\prime}}=x_{22^{\prime}} x_{21^{\prime}}, \\
{\left[x_{11^{\prime}}, x_{22^{\prime}}\right]+\left[x_{21^{\prime}}, x_{12^{\prime}}\right]=0} \\
x_{11^{\prime}} x_{21^{\prime}}=q^{-2} x_{21^{\prime}} x_{11^{\prime}}, \quad x_{12^{\prime}} x_{22^{\prime}}=q^{-2} x_{22^{\prime}} x_{12^{\prime}}, \\
x_{21^{\prime}} x_{12^{\prime}}=q^{2} x_{12^{\prime}} x_{21^{\prime}}
\end{gathered}
$$

and

$$
\begin{gathered}
y_{11^{\prime}} y_{21^{\prime}}=y_{21^{\prime}} y_{11^{\prime}}, \quad y_{12^{\prime}} y_{22^{\prime}}=y_{22^{\prime}} y_{12^{\prime}} \\
{\left[y_{11^{\prime}}, y_{22^{\prime}}\right]+\left[y_{12^{\prime}}, y_{21^{\prime}}\right]=0} \\
y_{11^{\prime}} y_{12^{\prime}}=q^{-2} y_{12^{\prime}} y_{11^{\prime}}, \quad y_{21^{\prime}} y_{22^{\prime}}=q^{-2} y_{22^{\prime}} y_{21^{\prime}}, \\
y_{12^{\prime}} y_{21^{\prime}}=q^{2} y_{21^{\prime}} y_{12^{\prime}}
\end{gathered}
$$

The determinant condition $\operatorname{det}_{q}(g)=1$ in the new variables becomes:

$$
x_{11^{\prime}} x_{22^{\prime}}-x_{12^{\prime}} x_{21^{\prime}}=x_{22^{\prime}} x_{11^{\prime}}-x_{21^{\prime}} x_{12^{\prime}}=\delta^{2}
$$

and

$$
y_{11^{\prime}} y_{22^{\prime}}-y_{21^{\prime}} y_{12^{\prime}}=y_{22^{\prime}} y_{11^{\prime}}-y_{12^{\prime}} y_{21^{\prime}}=\delta^{-2}
$$

Furthermore, the $\uparrow$ involution acts on these new variables in the following way:

$$
\begin{array}{llll}
x_{11^{\prime}}^{\dagger}=x_{22^{\prime}} & x_{12^{\prime}}^{\dagger}=-x_{21^{\prime}} & x_{21^{\prime}}^{\dagger}=-x_{12^{\prime}} & x_{22^{\prime}}^{\dagger}=x_{11^{\prime}} \\
y_{11^{\prime}}^{\dagger}=y_{22^{\prime}} & y_{12^{\prime}}^{\dagger}=-y_{21^{\prime}} & y_{21^{\prime}}^{\dagger}=-y_{12^{\prime}} & y_{22^{\prime}}^{\dagger}=y_{11^{\prime}}
\end{array}
$$


We will consider the following subalgebras of $\widetilde{S L}(2)_{q}$ :

$$
\left.\mathfrak{M}_{q}^{\mathrm{I}}=\mathbb{C}\left[x_{11^{\prime}}, x_{12^{\prime}}, x_{21^{\prime}}, x_{22^{\prime}}\right] /(21]-23\right]
$$

and

$$
\mathfrak{M}_{q}^{\mathrm{J}}=\mathbb{C}\left[y_{11^{\prime}}, y_{12^{\prime}}, y_{21^{\prime}}, y_{22^{\prime}}\right] /(24-26)
$$

regarding them as $q$-deformations of $\mathfrak{M}^{\mathrm{I}}$ and $\mathfrak{M}^{\mathrm{J}}$, the coordinate algebras corresponding to the affine subspaces $\mathbb{M}^{\mathrm{I}}$ and $\mathbb{M}^{\mathrm{J}}$ of the compactified complexified Minkowski space-time $\mathbb{M}$. Moreover, we introduce the $†$-algebras:

$$
S_{q}^{\mathrm{I}}=\left(\mathfrak{M}_{q}^{\mathrm{I}}, \dagger\right) \quad \text { and } \quad S_{q}^{\mathrm{J}}=\left(\mathfrak{M}_{q}^{\mathrm{J}}, \dagger\right)
$$

regarding them as $q$-deformations of $S^{4} \backslash\{\infty\}$ and $S^{4} \backslash\{0\}$, respectively.

Note also that $\mathfrak{M}_{q}^{\mathrm{I}}$ and $\mathfrak{M}_{q}^{\mathrm{J}}$ are isomorphic (as Hopf algebras) once the generators $\delta$ and $\delta^{-1}$ are adjoined. Let $X$ and $Y$ be again the matrices of generators as defined in (5); the map:

$$
\begin{aligned}
\eta: \mathfrak{M}_{q}^{\mathrm{I}}\left[\delta^{-1}\right] & \longrightarrow \mathfrak{M}_{q}^{\mathrm{J}}[\delta] \\
X & \mapsto \delta^{2} Y
\end{aligned}
$$

is an isomorphism. More explicitly, in coordinates:

$$
\begin{aligned}
x_{11^{\prime}} & \mapsto \delta^{2} y_{22^{\prime}} & x_{12^{\prime}} & \mapsto-\delta^{2} y_{12^{\prime}} \\
x_{21^{\prime}} & \mapsto-\delta^{2} y_{21^{\prime}} & x_{22^{\prime}} & \mapsto \delta^{2} y_{11^{\prime}}
\end{aligned}
$$

The inverse map $\eta^{-1}$ is given by $Y \mapsto X \delta^{-2}$. Moreover, $\eta\left(X^{\dagger}\right)=\eta(X)^{\dagger}$, so that $\eta\left(f^{\dagger}\right)=\eta(f)^{\dagger}$ for all $f \in \mathfrak{M}_{q}^{\mathrm{I}}\left[\delta^{-1}\right]$.

Geometrically, the algebras $\mathfrak{M}_{q}^{\mathrm{I}}\left[\delta^{-1}\right]$ and $\mathfrak{M}_{q}^{\mathrm{J}}[\delta]$ play the role of the intersection $\mathbb{M}^{\mathrm{IJ}}=\mathbb{M}^{\mathrm{I}} \cap \mathbb{M}^{\mathrm{J}}$, while $\eta$ plays the role of the gluing map $\tau$.

Furthermore, as in the case of the quantum group $G L(2)_{q}$, the above commutation relations can also be put in a compact form by means of an $R$-matrix. It is now convenient to consider the following matrix of generators:

$$
X=\left(\begin{array}{ll}
x_{11^{\prime}} & x_{12^{\prime}} \\
x_{21^{\prime}} & x_{22^{\prime}}
\end{array}\right) \quad Y=\left(\begin{array}{ll}
y_{11^{\prime}} & y_{12^{\prime}} \\
y_{21^{\prime}} & y_{22^{\prime}}
\end{array}\right)
$$


Notice that:

$$
X=Q^{-1} \delta T Q=Q T \delta Q^{-1} \quad \text { and } \quad Y=Q \delta^{-1} T Q^{-1}=Q^{-1} T \delta^{-1} Q
$$

where $Q$ was defined in (16). In order to write down the above commutation relations in matrix form, define $X_{1}=X \otimes \mathbf{1}, X_{2}=1 \otimes X$ and similarly $Q_{1}=Q \otimes \mathbf{1}, Q_{2}=\mathbf{1} \otimes Q$. Therefore:

$$
\begin{aligned}
X_{1} X_{2} & =\left(Q_{1}^{-1} \delta T_{1} Q_{1}\right)\left(Q_{2} T_{2} \delta Q_{2}^{-1}\right)=\left(Q_{1}^{-1} Q_{2}\right) \delta T_{1} T_{2} \delta\left(Q_{1} Q_{2}^{-1}\right)= \\
& =\left(Q_{1}^{-1} Q_{2} R_{12}^{-1} Q_{2} Q_{1}^{-1}\right) X_{2} X_{1}\left(Q_{2}^{-1} Q_{1} R_{12} Q_{1} Q_{2}^{-1}\right)
\end{aligned}
$$

Thus we define $R_{12}^{\mathrm{I}}=Q_{2}^{-1} Q_{1} R_{12} Q_{1} Q_{2}^{-1} ;$ more precisely:

$$
R_{12}^{\mathrm{I}}=\left(\begin{array}{cccc}
p^{-1} & 0 & 0 & 0 \\
0 & q^{-1} & p^{-1}-q & 0 \\
0 & p^{-1}-q^{-1} & q & 0 \\
0 & 0 & 0 & p^{-1}
\end{array}\right), \quad p=q^{ \pm 1}
$$

The commutation relations (21,23) can then be presented in matrix form:

$$
R_{12}^{\mathrm{I}} X_{1} X_{2}=X_{2} X_{1} R_{12}^{\mathrm{I}}
$$

Performing a similar calculation for the $y_{r s^{\prime}}$ variables, we obtain:

$$
R_{12}^{\mathrm{J}}=Q_{2} Q_{1}^{-1} R_{12} Q_{1}^{-1} Q_{2}=\left(\begin{array}{cccc}
p^{-1} & 0 & 0 & 0 \\
0 & q & p^{-1}-q & 0 \\
0 & p^{-1}-q^{-1} & q^{-1} & 0 \\
0 & 0 & 0 & p^{-1}
\end{array}\right), \quad p=q^{ \pm 1}
$$

with the commutation relations (24,26) being given by:

$$
R_{12}^{\mathrm{J}} Y_{1} Y_{2}=Y_{2} Y_{1} R_{12}^{\mathrm{J}}
$$




\section{Differential forms on quantum Minkowski space-time}

\subsection{Differential forms on Minkowski space-time}

Since $\mathbb{M}^{\mathrm{I}}$ and $\mathbb{M}^{\mathrm{J}}$ are affine spaces, their modules of differential forms are very simple to describe. Indeed, recall that:

$$
\mathfrak{M}^{\mathrm{I}}=\mathbb{C}\left[x_{11^{\prime}}, x_{12^{\prime}}, x_{21^{\prime}}, x_{22^{\prime}}\right]
$$

Therefore, the module of differential 1-forms is given by the free $\mathfrak{M}^{\mathrm{I}}$-module generated by $d x_{r s^{\prime}}$ :

$$
\Omega_{\mathfrak{M}^{\mathrm{I}}}^{1}=\mathfrak{M}^{\mathrm{I}}\left\langle d x_{r s^{\prime}}\right\rangle
$$

while the module of differential 2 -forms is given by the free $\mathfrak{M}^{\mathrm{I}}$-module generated by $d x_{r s^{\prime}} \wedge d x_{k l^{\prime}}$ :

$$
\Omega_{\mathfrak{M}^{\mathrm{I}}}^{2}=\Lambda^{2}\left(\Omega_{\mathfrak{M}^{\mathrm{I}}}^{1}\right)=\mathfrak{M}^{\mathrm{I}}\left\langle d x_{r s^{\prime}} \wedge d x_{k l^{\prime}}\right\rangle
$$

with $r, s, k, l=1,2$.

The action of the de Rham operator $d: \mathfrak{M}^{\mathrm{I}} \rightarrow \Omega_{\mathfrak{M}^{\mathrm{I}}}^{1}$ is given on the generators as $x_{r s^{\prime}} \mapsto d x_{r s^{\prime}}$, and it is then extended to the whole $\mathfrak{M}^{\mathrm{I}}$ by $\mathbb{C}$-linearity and the Leibnitz rule:

$$
d(f g)=g d f+f d g
$$

where $f, g \in \mathfrak{M}^{\mathrm{I}}$. One also defines the de Rham operator $d: \Omega_{\mathfrak{M}^{\mathrm{I}}}^{1} \rightarrow \Omega_{\mathfrak{M}^{\mathrm{I}}}^{2}$ on the generators as $f d x_{r s^{\prime}} \mapsto d f \wedge d x_{r s^{\prime}}$, also extending it by $\mathbb{C}$-linearity and the Leibnitz rule (35).

The modules of differential forms and de Rham operators over $\mathfrak{M}^{\mathrm{J}}$ are similarly described.

Now let $\Omega_{E^{4}}^{2}$ denote the bundle of 2-forms on Euclidean space $E^{4}$ with coordinates $\left\{x^{1}, x^{2}, x^{3}, x^{4}\right\}$. Recall that the Hodge operator $*: \Omega_{E^{4}}^{2} \rightarrow \Omega_{E^{4}}^{2}$ is defined as follows:

$$
* d x^{1} \wedge d x^{2}=d x^{3} \wedge d x^{4} \quad * d x^{3} \wedge d x^{4}=d x^{1} \wedge d x^{2}
$$




$$
\begin{array}{rl}
* d x^{1} \wedge d x^{3}=-d x^{2} \wedge d x^{4} & * d x^{2} \wedge d x^{4}=-d x^{1} \wedge d x^{3} \\
* d x^{1} \wedge d x^{4}=d x^{2} \wedge d x^{3} & * d x^{2} \wedge d x^{3}=d x^{1} \wedge d x^{4}
\end{array}
$$

We can then use the relation between Euclidean and twistor coordinates on $\mathbb{M}^{\mathrm{I}}=E^{4} \otimes \mathbb{C}$ given by (4) to express the action of the Hodge operator on $\Omega_{\mathfrak{M}^{\mathrm{I}}}^{2}$. One obtains:

$$
\begin{array}{lr}
* d x_{11^{\prime}} \wedge d x_{12^{\prime}}=d x_{11^{\prime}} \wedge d x_{12^{\prime}} & * d x_{11^{\prime}} \wedge d x_{21^{\prime}}=-d x_{11^{\prime}} \wedge d x_{21^{\prime}} \\
* d x_{11^{\prime}} \wedge d x_{22^{\prime}}=-d x_{12^{\prime}} \wedge d x_{21^{\prime}} & * d x_{12^{\prime}} \wedge d x_{21^{\prime}}=-d x_{11^{\prime}} \wedge d x_{22^{\prime}} \\
* d x_{12^{\prime}} \wedge d x_{22^{\prime}}=-d x_{12^{\prime}} \wedge d x_{22^{\prime}} & * d x_{21^{\prime}} \wedge d x_{22^{\prime}}=d x_{21^{\prime}} \wedge d x_{22^{\prime}}
\end{array}
$$

Clearly $*^{2}=1$, thus the complexified Hodge operator $*: \Omega_{\mathfrak{M}^{\mathrm{I}}}^{2} \rightarrow \Omega_{\mathfrak{M}^{\mathrm{I}}}^{2}$ induces a splitting of $\Omega_{\mathfrak{M}^{\mathrm{I}}}^{2}$ into two submodules corresponding to eigenvalues \pm 1 . More explicitly, we have:

$$
\Omega_{\mathfrak{M}^{\mathrm{I}}}^{2}=\Omega_{\mathfrak{M}^{\mathrm{I}}}^{2,+} \oplus \Omega_{\mathfrak{M}^{\mathrm{I}}}^{2,-}
$$

where

$$
\begin{aligned}
& \Omega_{\mathfrak{M}^{\mathrm{I}}}^{2,+}=\mathfrak{M}^{\mathrm{I}}\left\langle d x_{11^{\prime}} \wedge d x_{12^{\prime}}, d x_{21^{\prime}} \wedge d x_{22^{\prime}}, d x_{11^{\prime}} \wedge d x_{22^{\prime}}-d x_{12^{\prime}} \wedge d x_{21^{\prime}}\right\rangle \\
& \Omega_{\mathfrak{M}^{\mathrm{I}}}^{2,-}=\mathfrak{M}^{\mathrm{I}}\left\langle d x_{11^{\prime}} \wedge d x_{21^{\prime}}, d x_{12^{\prime}} \wedge d x_{22^{\prime}}, d x_{11^{\prime}} \wedge d x_{22^{\prime}}+d x_{12^{\prime}} \wedge d x_{21^{\prime}}\right\rangle
\end{aligned}
$$

Connection and curvature Let $E$ be a $\mathfrak{M}^{\mathrm{I}}$-module; a connection on $E$ is a $\mathbb{C}$-linear map:

$$
\nabla: E \rightarrow E \otimes_{\mathfrak{M}^{\mathrm{I}}} \Omega_{\mathfrak{M}^{\mathrm{I}}}^{1}
$$

satisfying the Leibnitz rule:

$$
\nabla(f \sigma)=\sigma \otimes d f+f \nabla \sigma
$$

where $f \in \mathfrak{M}^{\mathrm{I}}$ and $\sigma \in E$. The connection $\nabla$ also acts on 1-differentials, being defined as the additive map:

$$
\nabla: E \otimes_{\mathfrak{M}^{\mathrm{I}}} \Omega_{\mathfrak{M}^{\mathrm{I}}}^{1} \rightarrow E \otimes_{\mathfrak{M}^{\mathrm{I}}} \Omega_{\mathfrak{M}^{\mathrm{I}}}^{2}
$$


satisfying:

$$
\nabla(\sigma \otimes \omega)=\sigma \otimes d \omega+\omega \wedge \nabla \sigma
$$

where $\omega \in \Omega_{\mathfrak{M}^{\mathfrak{I}}}^{1}$.

Moreover, two connections $\nabla$ and $\nabla^{\prime}$ are said to be gauge equivalent if there is $g \in \operatorname{Aut}_{\mathfrak{M}^{\mathrm{I}}}(E)$ such that $\nabla=g^{-1} \nabla^{\prime} g$.

The curvature $F_{\nabla}$ is defined by the composition:

$$
E \stackrel{\nabla}{\longrightarrow} E \otimes_{\mathfrak{M}^{\mathrm{I}}} \Omega_{\mathfrak{M}^{\mathrm{I}}}^{1} \stackrel{\nabla}{\longrightarrow} E \otimes_{\mathfrak{M}^{\mathrm{I}}} \Omega_{\mathfrak{M}^{\mathrm{I}}}^{2}
$$

and it is easy to check that it is actually $\mathfrak{M}^{\mathrm{I}}$-linear. Therefore, $F_{\nabla}$ can be regarded as an element of $\operatorname{End}_{\mathfrak{M}^{\mathrm{I}}}(E) \otimes_{\mathfrak{M}^{\mathrm{I}}} \Omega_{\mathfrak{M}^{\mathrm{I}}}^{2}$. Furthermore, if $\nabla$ and $\nabla^{\prime}$ are gauge equivalent, then there is $g \in \operatorname{Aut}_{\mathfrak{M}^{\mathbb{I}}}(E)$ such that $F_{\nabla}=g^{-1} F_{\nabla^{\prime}} g$.

If $E$ is projective (hence free), any connection $\nabla$ can be encoded into a matrix $A \in \operatorname{End}_{\mathfrak{M}^{\mathrm{I}}}(E) \otimes_{\mathfrak{M}^{\mathrm{I}}} \Omega_{\mathfrak{M}^{\mathrm{I}}}^{1}$. Simply choose a basis $\left\{\sigma_{k}\right\}$ for $E$, and let $s=\sum a^{k} \sigma_{k}$, so that:

$$
\nabla s=\sum_{k}\left(\sigma_{k} \otimes d a^{k}+a^{k} \nabla \sigma_{k}\right)
$$

Thus it is enough to know how $\nabla$ acts on the basis $\left\{\sigma_{k}\right\}$ :

$$
\nabla \sigma_{k}=\sum_{l, \alpha} A_{k}^{l, \alpha} \sigma_{l} \otimes d x_{\alpha}
$$

and we define $A$ as the matrix with entries given by the 1-forms $\sum_{\alpha} A_{k}^{l, \alpha} d x_{\alpha}$.

Conversely, given $A \in \operatorname{End}_{\mathfrak{M}^{\mathbb{I}}}(E) \otimes_{\mathfrak{M}^{\mathfrak{I}}} \Omega_{\mathfrak{M}^{\mathbb{I}}}^{1}$, we define the connection:

$$
\nabla_{A} s=\sum_{k, l, \alpha}\left(\sigma_{k} \otimes d a^{k}+A_{k}^{l, \alpha} a^{k} \sigma_{l} \otimes d x_{\alpha}\right)
$$

\subsection{Differential forms on the quantum group $S U(2)_{q}$}

Let us now recall a few facts regarding the exterior algebra over the relevant quantum groups [30, 32, 34]. The module of 1-forms over the quantum group 
$G L(2)_{q}$, which we shall denote by $\Omega_{G L}^{1}$, is the $G L(2)_{q}$-bimodule generated by $d g_{r s^{\prime}}$ satisfying the following relations (written in matrix form):

$$
R_{12} T_{1} d T_{2}=d T_{2} T_{1}\left(R_{21}\right)^{-1}
$$

where $R_{12}$ is again the matrix (12) and $d T_{2}=\mathbf{1} \otimes d T$, with:

$$
d T=\left(\begin{array}{ll}
d g_{11^{\prime}} & d g_{12^{\prime}} \\
d g_{21^{\prime}} & d g_{22^{\prime}}
\end{array}\right)
$$

Similarly, the module of 2 -forms $\Omega_{G L}^{2}$, is the $G L(2)_{q}$-bimodule generated by $d g_{r s^{\prime}} \wedge d g_{k l^{\prime}}$, which satisfy the relations (written in matrix form):

$$
R_{12} d T_{1} \wedge d T_{2}=-d T_{2} \wedge d T_{1}\left(R_{21}\right)^{-1}
$$

where $d T_{1}=d T \otimes \mathbf{1}$. Furthermore, the commutation relations between $g_{m n^{\prime}}$ and $d g_{r s^{\prime}} \wedge d g_{k l^{\prime}}$ can be deduced from (38) and (39) as follows. Let $d T_{3}=$ $\mathbf{1} \otimes \mathbf{1} \otimes d T$. Denoting $R_{b a}=\left(R_{a b}\right)^{\mathrm{t}}$, we have $R_{12} T_{1} d T_{2} R_{21}=d T_{2} T_{1}$ and $R_{13} T_{1} d T_{3} R_{31}=d T_{3} T_{1}$. Therefore,

$$
\begin{aligned}
d T_{3} \wedge d T_{2} T_{1} & =d T_{3} \wedge\left(R_{12} T_{1} d T_{2} R_{21}\right)=R_{12} d T_{3} T_{1} \wedge d T_{2} R_{21}= \\
& =R_{12} R_{13} T_{1} d T_{3} R_{31} \wedge d T_{2} R_{21}=\left(R_{12} R_{13}\right) T_{1} d T_{3} \wedge d T_{2}\left(R_{12} R_{13}\right)^{\mathrm{t}}
\end{aligned}
$$

The noncommutative de Rham operators are given by their action on the generators as follows:

$$
\begin{array}{cc}
d: G L(2)_{q} \rightarrow \Omega_{G L}^{1} & d: \Omega_{G L}^{1} \rightarrow \Omega_{G L}^{2} \\
g_{r s^{\prime}} \mapsto d g_{r s^{\prime}} & g_{k l^{\prime}} d g_{r s^{\prime}} \mapsto d g_{k l^{\prime}} \wedge d g_{r s^{\prime}}
\end{array}
$$

This is then extended to the whole $G L(2)_{q}$ and $\Omega_{G L}^{1}$ by $\mathbb{C}$-linearity and the Leibnitz rule:

$$
d\left(f_{1} f_{2}\right)=d f_{1} f_{2}+f_{1} d f_{2}
$$

for all $f_{1}, f_{2} \in G L(2)_{q}$.

The modules $\Omega_{G L}^{1}$ and $\Omega_{G L}^{2}$ also have natural involutions, extended from $\dagger$ in a natural way, namely:

$$
\left(f d g_{r s^{\prime}}\right)^{\dagger}=d g_{r s^{\prime}}^{\dagger} f^{\dagger}
$$




$$
\left(f d g_{r s^{\prime}} \wedge d g_{k l^{\prime}}\right)^{\dagger}=-d g_{k l^{\prime}}^{\dagger} \wedge d g_{r s^{\prime}}^{\dagger} f^{\dagger}
$$

To get the modules of forms on $S L(2)_{q}$ it is enough to take the quotient by the appropriate relations:

$$
\Omega_{S L}^{1}=\Omega_{G L}^{1} / \begin{gathered}
\operatorname{det}_{q} T=1 \\
d\left(\operatorname{det}_{q} T\right)=0
\end{gathered} \quad \text { and } \quad \Omega_{S L}^{2}=\Lambda^{2}\left(\Omega_{S L}^{1}\right)
$$

Finally, modules of forms on $S U(2)_{q}$ are then defined as the pairs:

$$
\Omega_{S U}^{k}=\left(\Omega_{S L}^{k}, \dagger\right), \quad k=1,2
$$

It is also important for our purposes to describe the modules of 1- and 2 -forms on the extended quantum group $\widetilde{S L}(2)_{q}$. To do that, we add the generators $\delta$ and $d \delta$ satisfying the relations (written in matrix form):

$$
\delta d T Q^{2}=Q^{2} d T \delta \quad \text { and } \quad d \delta d T Q^{2}=-Q^{2} d T d \delta
$$

We extend the involution $\dagger$ to $\Omega_{\widetilde{S L}}^{k}$ by declaring that $(d \delta)^{\dagger}=d \delta$; thus we have:

$$
\Omega_{\widetilde{S U}}^{k}=\left(\Omega_{\widetilde{S L}}^{k}, \dagger\right), \quad k=1,2
$$

Let us now introduce the noncommutative analogue of the Hodge operator on $\Omega_{G L}^{2}$. This will serve as a model on our Definition of self-dual and antiself-dual 2-forms on quantum space-time.

Recall that $R_{12}$ denotes the $R$-matrix (12) for $G L(2)_{q}$. Define:

$$
P^{+}=\frac{\hat{R}_{12}+p \mathbf{1}}{p+p^{-1}} \quad \text { and } \quad P^{-}=\frac{-\hat{R}_{12}+p^{-1} \mathbf{1}}{p+p^{-1}}
$$

Clearly, $P^{+}+P^{-}=\mathbf{1}$. Moreover, using the Hecke relations (14), one easily checks that $\left(P^{+}\right)^{2}=P^{+}$and $\left(P^{-}\right)^{2}=P^{-}$. Now the Hodge $*$-operator is defined on the generators of $\Omega_{G L}^{2}$ by:

$$
* d T_{1} \wedge d T_{2}=\left(P^{+}-P^{-}\right) d T_{1} \wedge d T_{2}
$$




\subsection{Differential forms on quantum Minkowski space- time}

In analogy with the classical case, we define the module of 1-forms over the algebra $\mathfrak{M}_{q}^{\mathrm{I}}$, which we shall denote by $\Omega_{\mathfrak{M}_{q}^{\mathrm{I}}}^{1}$, as the $\mathfrak{M}_{q}^{\mathrm{I}}$-bimodule generated by:

$$
d X=\left(\begin{array}{ll}
d x_{11^{\prime}} & d x_{12^{\prime}} \\
d x_{21^{\prime}} & d x_{22^{\prime}}
\end{array}\right)=Q^{-1} \delta d T Q=Q d T \delta Q^{-1}
$$

Moreover, the generators $d x_{r s^{\prime}}$ satisfy the following relations (written in matrix form):

$$
\begin{aligned}
X_{1} d X_{2} & =\left(Q_{1}^{-1} Q_{2}\right) \delta T_{1} d T_{2} \delta\left(Q_{1} Q_{2}^{-1}\right)=\left(Q_{1}^{-1} Q_{2}\right) \delta R_{12}^{-1} d T_{2} T_{1} R_{21}^{-1} \delta\left(Q_{1} Q_{2}^{-1}\right)= \\
& =\left(Q_{1}^{-1} Q_{2} R_{12}^{-1} Q_{2} Q_{1}^{-1}\right) d X_{2} X_{1}\left(Q_{2}^{-1} Q_{1} R_{21}^{-1} Q_{1} Q_{2}^{-1}\right)
\end{aligned}
$$

where $R_{12}$ is again the matrix (12) and $d X_{2}=1 \otimes d X$. Thus using the $R$-matrix for $\mathfrak{M}_{q}^{\mathrm{I}}(33)$ and defining $R_{21}^{\mathrm{I}}=Q_{1}^{-1} Q_{2} R_{21} Q_{1}^{-1} Q^{2}$, we obtain:

$$
R_{12}^{\mathrm{I}} X_{1} d X_{2}=d X_{2} X_{1}\left(R_{21}^{\mathrm{I}}\right)^{-1}
$$

Similarly, the module of 2 -forms $\Omega_{\mathfrak{M}_{q}^{\mathrm{I}}}^{2}$, is the $\mathfrak{M}_{q}^{\mathrm{I}}$-bimodule generated by $d x_{r s^{\prime}} \wedge d x_{k l^{\prime}}$ satisfying the relations below (written in matrix form), which can be deduced from (42):

$$
R_{12}^{\mathrm{I}} d X_{1} \wedge d X_{2}=-d X_{2} \wedge d X_{1}\left(R_{21}^{\mathrm{I}}\right)^{-1}
$$

where $d X_{1}=d X \otimes 1$.

Performing the same calculations for $\mathfrak{M}_{q}^{\mathrm{J}}$, we conclude that $\Omega_{\mathfrak{M}_{q}^{\mathrm{J}}}^{1}$ and $\Omega_{\mathfrak{M}_{q}^{j}}^{2}$ are the $\mathfrak{M}_{q}^{\mathrm{J}}$-bimodules generated by $d y_{r s^{\prime}}$ and $d y_{r s^{\prime}} \wedge d y_{k l^{\prime}}$, respectively, satisfying the following relations (written in matrix form):

$$
R_{12}^{\mathrm{J}} Y_{1} d Y_{2}=d Y_{2} Y_{1}\left(R_{21}^{\mathrm{J}}\right)^{-1} \quad \text { and } \quad R_{12}^{\mathrm{J}} d Y_{1} \wedge d Y_{2}=-d Y_{2} \wedge d Y_{1}\left(R_{21}^{\mathrm{J}}\right)^{-1}
$$

where $R_{12}^{\mathrm{J}}$ is the $R$-matrix for $\mathfrak{M}_{q}^{\mathrm{J}}(\underline{34}), R_{21}^{\mathrm{J}}=Q_{2}^{-1} Q_{1} R_{21} q_{1} Q_{2}^{-1}$ and

$$
d Y=\left(\begin{array}{ll}
d y_{11^{\prime}} & d y_{12^{\prime}} \\
d y_{21^{\prime}} & d y_{22^{\prime}}
\end{array}\right)=Q \delta^{-1} d T Q^{-1}=Q^{-1} d T \delta^{-1} Q
$$


with $d Y_{1}=d Y \otimes \mathbf{1}$ and $d Y_{2}=\mathbf{1} \otimes d Y$.

We now introduce the concept of anti-self-duality of quantum 2-forms over the quantum Minkowski space-time. Notice that the matrices $R_{12}^{\mathrm{I}}, R_{21}^{\mathrm{I}}$ and $R_{12}^{\mathrm{J}}, R_{21}^{\mathrm{J}}$ also satisfy Hecke relations (recall that $P$ denotes the permutation matrix):

$$
R_{12}^{\mathrm{I}}-\left(R_{21}^{\mathrm{I}}\right)^{-1}=\left(p^{-1}-p\right) P \quad \text { and } \quad R_{12}^{\mathrm{J}}-\left(R_{21}^{\mathrm{J}}\right)^{-1}=\left(p^{-1}-p\right) P
$$

Moreover, defining $\hat{R}_{12}^{\mathrm{I}}=P R_{12}^{\mathrm{I}}$ and $\hat{R}_{12}^{\mathrm{J}}=P R_{12}^{\mathrm{J}}$, we obtain:

$$
\left(\hat{R}_{12}^{\mathrm{I}}\right)^{2}=\mathbf{1}+\left(p^{-1}-p\right) \hat{R}_{12}^{\mathrm{I}} \quad \text { and } \quad\left(\hat{R}_{12}^{\mathrm{J}}\right)^{2}=\mathbf{1}+\left(p^{-1}-p\right) \hat{R}_{12}^{\mathrm{J}}
$$

in analogy with (14). Therefore we can proceed as in the case of $\widetilde{G L}(2)_{q}$ discussed above and define the projectors:

$$
\begin{aligned}
P^{\mathrm{I}+} & =\frac{\hat{R}_{12}^{\mathrm{I}}+p \mathbf{1}}{p+p^{-1}} \quad \text { and } \quad P^{\mathrm{I}-}=\frac{-\hat{R}_{12}^{\mathrm{I}}+p^{-1} \mathbf{1}}{p+p^{-1}} \\
P^{\mathrm{J}+} & =\frac{\hat{R}_{12}^{\mathrm{J}}+p \mathbf{1}}{p+p^{-1}} \quad \text { and } \quad P^{\mathrm{J}-}=\frac{-\hat{R}_{12}^{\mathrm{J}}+p^{-1} \mathbf{1}}{p+p^{-1}}
\end{aligned}
$$

Now we define the Hodge operator on $\Omega_{\mathfrak{M}_{q}^{I}}^{2}$ (in matrix form):

$$
* d X_{1} \wedge d X_{2}=\left(P^{\mathrm{I}+}-P^{\mathrm{I}-}\right) d X_{1} \wedge d X_{2}
$$

Since $*^{2}=\mathbf{1}$, the module $\Omega_{\mathfrak{M}_{q}^{\mathrm{I}}}^{2}$ can be decomposed into two submodules corresponding to eigenvalues \pm 1 . Denote such submodules by $\Omega_{\mathfrak{M}_{q}^{\mathrm{I}}}^{2,+}$ and $\Omega_{\mathfrak{M}_{q}^{\mathrm{I}}}^{2,-}$.

In order to compare with the commutative case, it is instructive to write down their bases, which are given by the entries of the matrices $P^{\mathrm{I}+} d X_{1} \wedge$ $d X_{2}$ and $P^{\mathrm{I}-} d X_{1} \wedge d X_{2}$. After applying the commutation relations (39), we conclude that:

$$
\begin{aligned}
\Omega_{\mathfrak{M}_{q}^{I}}^{2,+} & =\mathfrak{M}_{q}^{\mathrm{I}}\left\langle d x_{11^{\prime}} \wedge d x_{12^{\prime}}, d x_{21^{\prime}} \wedge d x_{22^{\prime}}, d x_{11^{\prime}} \wedge d x_{22^{\prime}}-d x_{12^{\prime}} \wedge d x_{21^{\prime}}\right\rangle \\
\Omega_{\mathfrak{M}_{q}^{\mathrm{I}}}^{2,-} & =\mathfrak{M}_{q}^{\mathrm{I}}\left\langle d x_{11^{\prime}} \wedge d x_{21^{\prime}}, d x_{12^{\prime}} \wedge d x_{22^{\prime}}, d x_{11^{\prime}} \wedge d x_{22^{\prime}}+d x_{12^{\prime}} \wedge d x_{21^{\prime}}\right\rangle
\end{aligned}
$$

in complete analogy with the commutative case.

Finally, replacing all the I's by J's, we define the Hodge operator on $\Omega_{\mathfrak{M}_{q}^{J}}^{2}$ as well:

$$
* d Y_{1} \wedge d Y_{2}=\left(P^{\mathrm{J}+}-P^{\mathrm{J}-}\right) d Y_{1} \wedge d Y_{2}
$$


Connection and curvature Let $E$ be a right $\mathfrak{M}_{q}^{\mathrm{I}}$-module. In analogy with the commutative case, a connection on $E$ is a $\mathbb{C}$-linear map:

$$
\nabla: E \rightarrow E \otimes_{\mathfrak{M}_{q}^{I}} \Omega_{\mathfrak{M}_{q}^{I}}^{1}
$$

satisfying the Leibnitz rule:

$$
\nabla(\sigma f)=\sigma \otimes d f+\nabla(\sigma) f
$$

where $f \in \mathfrak{M}_{q}^{\mathrm{I}}$ and $\sigma \in E$. The connection $\nabla$ also acts on 1-forms, being defined as the $\mathbb{C}$-linear map:

$$
\nabla: E \otimes_{\mathfrak{M}_{q}^{\mathrm{I}}} \Omega_{\mathfrak{M}_{q}^{\mathrm{I}}}^{1} \rightarrow E \otimes_{\mathfrak{M}_{q}^{\mathrm{I}}} \Omega_{\mathfrak{M}_{q}^{\mathrm{I}}}^{2}
$$

satisfying:

$$
\nabla(\sigma \otimes \omega)=\sigma \otimes d \omega+\nabla \sigma \wedge \omega
$$

where $\omega \in \Omega_{\mathfrak{M}_{q}^{\mathrm{I}}}^{1}$.

Moreover, two connections $\nabla$ and $\nabla^{\prime}$ are said to be gauge equivalent if there is $g \in \operatorname{Aut}_{\mathfrak{M}_{q}^{\mathrm{I}}}(E)$ such that $\nabla=g^{-1} \nabla^{\prime} g$.

The curvature $F_{\nabla}$ is defined by the composition:

$$
E \stackrel{\nabla}{\longrightarrow} E \otimes_{\mathfrak{M}_{q}^{\mathrm{I}}} \Omega_{\mathfrak{M}_{q}^{\mathrm{I}}}^{1} \stackrel{\nabla}{\longrightarrow} E \otimes_{\mathfrak{M}_{q}^{\mathrm{I}}} \Omega_{\mathfrak{M}_{q}^{\mathrm{I}}}^{2}
$$

and it is easy to check that $F_{\nabla}$ is actually right $\mathfrak{M}_{q}^{\mathrm{I}}$-linear. Therefore, $F_{\nabla}$ can be regarded as an element of $\operatorname{End}_{\mathfrak{M}_{q}^{\mathrm{I}}}(E) \otimes_{\mathfrak{M}_{q}^{\mathrm{I}}} \Omega_{\mathfrak{M}_{q}^{\mathrm{I}}}^{2}$. Furthermore, if $\nabla$ and $\nabla^{\prime}$ are gauge equivalent, then there is $g \in \operatorname{Aut}_{\mathfrak{M}^{\mathrm{I}}}(E)$ such that $F_{\nabla}=g^{-1} F_{\nabla^{\prime}} g$. A connection $\nabla$ is said to be anti-self-dual if $F_{\nabla} \in \operatorname{End}_{\mathfrak{M}_{q}^{\mathrm{I}}}(E) \otimes_{\mathfrak{M}_{q}^{\mathrm{I}}} \Omega_{\mathfrak{M}_{q}^{\mathrm{I}}}^{2,-}$.

Finally, if $E$ is projective (though not necessarily free in this case), any connection $\nabla$ can be encoded into the connection matrix $A \in \operatorname{End}_{\mathfrak{M}_{q}^{\mathrm{I}}}\left(\oplus^{n} \mathfrak{M}_{q}^{\mathrm{I}}\right) \otimes_{\mathfrak{M}_{q}^{\mathrm{I}}}$ $\Omega_{\mathfrak{M}_{q}^{\mathfrak{I}}}^{1}$ (where $n$ is the rank of $E$ ) in the following way. First, we recall the following basic result from algebra (see e.g. [16]):

Theorem 1 (Dual basis theorem). A finitely generated right $R$-module $M$ is projective of rank $n$ if and only if there are elements $\sigma_{k} \in M$ and $\rho^{k} \in M^{\vee}=\operatorname{Hom}_{R}(M, R)$ for $k=1, \ldots, n$ such that $m=\sum_{k} \sigma_{k} \rho^{k}(m)$, for any $m \in M$. 
Let $\left\{\sigma_{k}, \rho^{k}\right\}$ be a dual basis for $E$, so that any $s \in E$ can be written as $s=\sum_{k} \sigma_{k} \rho^{k}(s)$; applying the Leibnitz rule, we get:

$$
\nabla s=\sum_{k}\left(\sigma_{k} \otimes d\left(\rho^{k}(s)\right)+\nabla\left(\sigma_{k}\right) \rho^{k}(s)\right)
$$

Thus, as in the commutative case, it is enough to know how $\nabla$ acts on $\left\{\sigma_{k}\right\}$; we set:

$$
\nabla \sigma_{k}=\sum_{l} \sigma_{l} A_{k}^{l}, \quad \text { with } \quad A_{k}^{l}=\rho^{l} \otimes \mathbf{1}\left(\nabla \sigma_{k}\right) \in \Omega_{\mathfrak{M}_{q}^{\mathrm{I}}}^{1}
$$

and we define $A$ as the matrix with entries given by the 1-forms $A_{k}^{l}$. Clearly, $A$ depends on the choice of dual basis; changing the dual basis amounts to a change of gauge for $A$.

Conversely given a matrix $A \in \operatorname{End}_{\mathfrak{M}_{q}^{\mathrm{I}}}\left(\oplus^{n} \mathfrak{M}_{q}^{\mathrm{I}}\right) \otimes_{\mathfrak{M}_{q}^{\mathrm{I}}} \Omega_{\mathfrak{M}_{q}^{\mathrm{I}}}^{1}$, we define the connection:

$$
\nabla_{A} s=\sum_{l, k} \sigma_{k} \otimes d\left(\rho^{k}(s)\right)+\sigma_{l} A_{k}^{l} \rho^{k}(s)
$$

\section{Construction of quantum instantons}

\subsection{Classical instantons and the ADHM data}

As we mentioned in Introduction, solutions of the classical ASDYM equations can be constructed from certain linear data, so-called ADHM data. We will now briefly review some relevant facts regarding this correspondence.

Recall that the ASDYM equation can be defined for a complex vector bundle $E$ over any four dimensional Riemannian manifold $X$, provided with a connection $\nabla$. This connection is said to be anti-self-dual if the corresponding curvature 2-form $F_{\nabla}$ satisfies the equation ( $*$ is the Hodge operator on 2forms):

$$
* F_{\nabla}=-F_{\nabla}
$$

i.e., if $F_{\nabla} \in \operatorname{End}(E) \otimes \Omega_{X}^{2,-}$. An ASD connection is usually called an instanton 
if the integral:

$$
c=\frac{1}{8 \pi^{2}} \int_{X} \operatorname{Tr}\left(F_{\nabla} \wedge F_{\nabla}\right)
$$

converges. If $X$ is a compact manifold, $c$ is actually an integer, so-called instanton number or charge, and coincides with the second Chern class of $E$. In view of the symmetry between self-dual and anti-self-dual connections (they only differ by the choice of orientation on $X$ ), one can assume without loss of generality that $c>0$. Furthermore, a framing for an instanton on $X$ at a point $p \in X$ is the choice of an isomorphism $E_{p} \simeq \mathbb{C}^{n}$, where $E_{p}$ denotes the fiber of $E$ at $p$.

In the celebrated paper [1], Atiyah, Drinfeld, Hitchin and Manin constructed a class of ASD connections on the simplest compact four dimensional manifold, namely the four dimensional sphere $S^{4}$. They also proved that their construction is complete in the sense that any ASD connection on $S^{4}$ is gauge equivalent to a connection constructed by them from a certain algebraic data that depends on the rank $n$ of the vector bundle $E$ and the instanton number $c$.

More precisely, let $V$ and $W$ be Hermitian vector spaces of dimensions $c$ and $n$, respectively. The algebraic data of Atiyah, Drinfeld, Hitchin and Manin consists of four linear operators:

$$
B_{1}, B_{2} \in \operatorname{End}(V), \quad i \in \operatorname{Hom}(W, V), \quad j \in \operatorname{Hom}(V, W)
$$

satisfying the following linear relations ( $†$ denotes Hermitian conjugation):

$$
\begin{aligned}
{\left[B_{1}, B_{2}\right]+i j } & =0 \\
{\left[B_{1}, B_{1}^{\dagger}\right]+\left[B_{2}, B_{2}^{\dagger}\right]+i i^{\dagger}-j^{\dagger} j } & =0
\end{aligned}
$$

plus a regularity condition which we describe below.

The ADHM data admits a natural action of the unitary group $U(V)$ :

$$
g\left(B_{1}, B_{2}, i, j\right)=\left(g B_{1} g^{-1}, g B_{2} g^{-1}, g i, j g^{-1}\right), \quad g \in U(V)
$$


We say that the ADHM datum $\left(B_{1}, B_{2}, i, j\right)$ is regular if its stabilizer subgroup is trivial. Equivalently, $\left(B_{1}, B_{2}, i, j\right)$ is regular if and only if it satisfies the following two conditions:

- stability: there is no proper subspace $S \subset V$ such that $B_{k}(S) \subset S$ $(k=1,2)$ and $i(W) \subset S$;

- costability: there is no proper subspace $S \subset V$ such that $B_{k}(S) \subset S$ $(k=1,2)$ and $S \subset \operatorname{ker} j$.

The following lemma is probably well known to the experts:

Lemma 2. Suppose that $\left(B_{1}, B_{2}, i, j\right)$ satisfies the ADHM equations (49) and (50). Then $\left(B_{1}, B_{2}, i, j\right)$ is stable if and only if it is also costable.

Proof. If $\left(B_{1}, B_{2}, i, j\right)$ is not stable, then by duality on $V$ there is a proper subspace $S^{\perp} \subset V$ such that $B_{k}^{\dagger}\left(S^{\perp}\right) \subset S^{\perp}$ and $S^{\perp} \subset$ ker $i^{\dagger}$. So restricting (50) to $S^{\perp}$ and taking the trace, we conclude that $\operatorname{Tr}\left(\left.j^{\dagger} j\right|_{S^{\perp}}\right)=0$. Hence $S^{\perp} \subset \operatorname{ker} j$, and $\left(B_{1}, B_{2}, i, j\right)$ is not costable.

Conversely, $\left(B_{1}, B_{2}, i, j\right)$ is not costable, take $S^{\perp} \subset V$ nonempty such that $B_{k}^{\dagger}\left(S^{\perp}\right) \subset S^{\perp}$ and $S^{\perp} \subset \operatorname{ker} j$. Restricting (150) to $S^{\perp}$ and taking the trace we conclude that $\operatorname{Tr}\left(\left.i i^{\dagger}\right|_{S^{\perp}}\right)=0$. Hence $S^{\perp} \subset$ ker $i^{\dagger}$, and dualizing equation (49) we see that $\left.\left[B_{1}^{\dagger}, B_{2}^{\dagger}\right]\right|_{S^{\perp}}=0$. Thus $S$ is a proper subspace of $V$ such that $B_{k}(S) \subset S$ and $i(W) \subset S$, contradicting stability.

We denote the space of regular orbits by:

$$
\mathcal{M}^{\mathrm{reg}}(n, c)=\left\{\left(B_{1}, B_{2}, i, j\right) \mid(\underline{49}),(50)\right\} / U(V)
$$

The main result of [1] is the following:

Theorem 3. The space $\mathcal{M}^{\mathrm{reg}}(n, c)$ is the moduli space of framed instantons of rank $n$ and charge $c$ on $S^{4}$. In other words, there is a bijection between points of $\mathcal{M}^{\mathrm{reg}}(n, c)$ and gauge equivalence classes of framed ASD connections of rank $n$ and charge $c$. 


\subsection{Quantum Haar measure and duality}

For later reference, we now explain the notion of duality on free $\mathfrak{M}_{q}^{\mathrm{I}}$ - and $\mathfrak{M}_{q}^{\mathrm{J}}$-modules. We also now specialize the formal parameter $q$ to a positive real number for the remainder of this section.

Recall that a Haar functional on a Hopf algebra $\mathcal{A}$ is a linear functional $H: \mathcal{A} \rightarrow \mathbb{C}$ satisfying the following conditions:

- bi-invariance: $(H \otimes \mathbf{1}) \circ \Delta(a)=(\mathbf{1} \otimes H) \circ \Delta(a)=H(a)$;

- antipode invariance: $H(\gamma(a))=H(a)$;

- normalization: $H(1)=1$.

Theorem 4. There is a unique Haar functional $H$ on the quantum group $S L(2)_{q}$, which induces a positive definite Hermitian form on $S U(2)_{q}$, namely:

$$
\left(g_{1}, g_{2}\right)=H\left(f^{\dagger} g\right), \quad g_{1}, g_{2} \in S L(2)_{q}
$$

Proof. The existence and uniqueness of the Haar functional was established in [14, 20, 33. The verification of the fact that $H$ induces a Hermitian form also can be found in these papers.

Now let $U$ be a finite dimensional Hermitian vector space, and let $(\cdot, \cdot)$ denote its Hermitian inner product, which is chosen to be conjugate linear in the first argument. We define the pairing:

$$
\begin{aligned}
(\cdot, \cdot): U \otimes \mathfrak{M}_{q}^{\mathrm{I}} \times U \otimes \mathfrak{M}_{q}^{\mathrm{I}} & \rightarrow \mathfrak{M}_{q}^{\mathrm{I}} \\
\left(v_{1} \otimes f_{1}, v_{2} \otimes f_{2}\right) & =\left(v_{1}, v_{2}\right) f_{1}^{\dagger} f_{2}
\end{aligned}
$$

From a geometrical point of view, the pairing above plays the role of a Her-

mitian metric on a (trivial) vector bundle over the Euclidean $\mathbb{R}^{4}$ : the pairing of two sections of the bundle gives a function on $\mathbb{R}^{4}$.

Proposition 5. The pairing (54) is non-degenerate, i.e.:

$$
(\sigma, \sigma)=0 \Leftrightarrow \sigma=0
$$


Proof. We extend the Haar functional to $\widetilde{S L}(2)_{q}$ as the homomorphism $\tilde{H}$ : $\widetilde{S L}(2)_{q} \rightarrow \mathbb{C}\left[\delta, \delta^{-1}\right]$ such that $(n \in \mathbb{Z})$ :

$$
\tilde{H}\left(\delta^{n} g\right)=\delta^{n} H(g)
$$

Now let $\left\{v_{k}\right\}$ be an orthonormal basis for $U$, and take $\sigma=\sum_{k} v_{k} \otimes f_{k}$, where $f_{k} \in \mathfrak{M}_{q}^{\mathrm{I}}$; we can assume that $f_{k}=\sum_{\alpha \geq 0} \delta^{\alpha} f_{k \alpha}$, with $f_{k \alpha} \in S L_{q}(2)$.

Then $0=\tilde{H}((\sigma, \sigma))$ forces, by the non degeneracy of $H, f_{k 0}=0$, i.e. $\sigma=\sigma_{1} \delta$, for some $\sigma_{1}$. Then $0=(\sigma, \sigma)=\delta\left(\sigma_{1}, \sigma_{1}\right) \delta$, and $\left(\sigma_{1}, \sigma_{1}\right)=0$ by the invertibility of $\delta$ in $S L_{q}(2)$. By iterating this procedure we get that $\sigma=0$.

Now consider $F_{l}=U_{l} \otimes \mathfrak{M}_{q}^{\mathrm{I}}$ as right $\mathfrak{M}_{q}^{\mathrm{I}}$-modules, where $U_{l}$ are Hermitian vector spaces, $l=1,2$. Let $F_{l}^{\dagger}$ denote the set of all maps $\mu: F_{l} \rightarrow \mathfrak{M}_{q}^{\mathrm{I}}$ such that $\mu(\sigma x)=\mu(\sigma) x$, for all $\sigma \in F_{l}$ and $x \in \mathfrak{M}_{q}^{\mathrm{I}}$, with the structure of a right $\mathfrak{M}_{q}^{\mathrm{I}}$-module defined by:

$$
(\mu x)(\sigma)=x^{\dagger} \mu(\sigma) \quad \forall \sigma \in F_{l}
$$

By Proposition 5, the map:

$$
\begin{aligned}
\mathcal{I}_{l}: F_{l} & \longrightarrow F_{l}^{\dagger} \\
\sigma & \mapsto(\sigma, \cdot)
\end{aligned}
$$

is injective, and it is easy to see that $\mathcal{I}$ must also be surjective. Moreover, notice that

$$
\mathcal{I}(\sigma x)=x^{\dagger} \mathcal{I}(\sigma)
$$

In other words, the map $\mathcal{I}$ provides an identification between a free $\mathfrak{M}_{q^{-}}^{\mathrm{I}}$ module and its dual. In particular, one can also regard $\dagger$ as an involution on $F_{l}$, defined as follows:

$$
(v \otimes f)^{\dagger}=\bar{v} \otimes f^{\dagger}
$$

and it is easy to see that $(\sigma x)^{\dagger}=x^{\dagger} \sigma^{\dagger}$ (with this equality being understood in terms of the bimodule structure of the free modules $F_{l}$ ). 
Any map $\mathcal{L}: F_{1} \rightarrow F_{2}$ satisfying $\mathcal{L}(\sigma x)=\mathcal{L}(\sigma) x$ induces a dual map $\mathcal{L}^{\vee}: F_{2}^{\dagger} \rightarrow F_{1}^{\dagger}$ in the usual way:

$$
\mathcal{L}^{\vee}(\varphi)=\varphi \circ \mathcal{L}
$$

Finally, the identification (55) yields a map $\mathcal{L}^{\dagger}=\mathcal{I}_{1}^{-1} \mathcal{L}^{\vee} \mathcal{I}_{2}: F_{2} \rightarrow F_{1}$ with the property:

$$
\left(\mathcal{L}^{\dagger} \sigma_{1}, \sigma_{2}\right)=\left(\sigma_{1}, \mathcal{L} \sigma_{2}\right) \quad \sigma_{l} \in F_{l}
$$

In particular, let us consider $\mathcal{L}=L \otimes x$, where $L \in \operatorname{Hom}\left(U_{1}, U_{2}\right)$ and $x$ means multiplication by $x \in \mathfrak{M}_{q}^{\mathrm{I}}$ in the left. Then the definition (154) immediately implies that $\mathcal{L}^{\dagger}=L^{\dagger} \otimes x^{\dagger}$.

Proposition 6. If $\mathcal{L}: F_{1} \rightarrow F_{2}$ is injective, then

$$
F_{2}=\operatorname{Im} \mathcal{L} \oplus \operatorname{ker} \mathcal{L}^{\dagger}
$$

Proof. Since $\mathcal{L}$ is injective, it is easy to see that $\operatorname{Im} \mathcal{L}$ is a free submodule of $F_{2}$. Let $N \subset F_{2}$ be such that $F_{2}=\operatorname{Im} \mathcal{L} \oplus N$. Define:

$$
(\operatorname{Im} \mathcal{L})^{0}=\left\{\psi \in F_{2}^{\dagger} \mid \psi(\nu)=0 \forall \nu \in \operatorname{Im} \mathcal{L}\right\}
$$

One can then show that $N^{\dagger} \simeq(\operatorname{Im} \mathcal{L})^{0} \simeq \operatorname{ker} \mathcal{L}^{\vee}$. The desired decomposition now follows from the identification $N \simeq N^{\dagger}$.

\subsection{Construction of quantum instantons}

First of all, we must explain precisely what we mean by a quantum instanton on the quantum affine Minkowski space.

Definition. A quantum instanton on $S_{q}^{\mathrm{I}}$ is a triple $(E, \nabla, \dagger)$ consisting of:

- a finitely generated, projective right $\mathfrak{M}_{q}^{\mathrm{I}}$-module $E$ equipped with an involution $\dagger: E \rightarrow E$ satisfying $(\sigma x)^{\dagger}=x^{\dagger} \sigma^{\dagger}$ for all $x \in \mathfrak{M}_{q}^{\mathrm{I}}$ and $\sigma \in E$; 
- anti-self-dual connection $\nabla: E \rightarrow E \otimes \Omega_{\mathfrak{M}_{q}^{\mathrm{I}}}^{1}$ which is compatible with the involution $\dagger$, i.e. $\nabla \dagger=\dagger \nabla$.

Quantum instantons on $S_{q}^{\mathrm{J}}$ are similarly defined. Moreover, we also define the notion of consistency between quantum instantons on $S_{q}^{\mathrm{I}}$ and $S_{q}^{\mathrm{J}}$ :

Definition. Quantum instantons $\left(E_{\mathrm{I}}, \nabla_{\mathrm{I}}, \dagger_{\mathrm{I}}\right)$ on $S_{q}^{\mathrm{I}}$ and $\left(E_{\mathrm{J}}, \nabla_{\mathrm{J}}, \dagger_{\mathrm{J}}\right)$ on $S_{q}^{\mathrm{J}}$ are said to be consistent if:

- there is an isomorphism

$$
\Gamma: E_{\mathrm{I}}\left[\delta^{-1}\right] \rightarrow E_{\mathrm{J}}[\delta]
$$

such that $\Gamma(\sigma f)=\Gamma(\sigma) \eta(f)$, for all $\sigma \in E_{\mathrm{I}}\left[\delta^{-1}\right]$ and $f \in \mathfrak{M}_{q}^{\mathrm{I}}\left[\delta^{-1}\right]$;

- $\nabla_{\mathrm{J}} \Gamma=\Gamma \nabla_{\mathrm{I}}$

- $\dagger_{\mathrm{J}} \Gamma=\Gamma \dagger_{\mathrm{I}}$.

Recall that $\eta$ is the isomorphism $\mathfrak{M}_{q}^{\mathrm{I}}\left[\delta^{-1}\right] \rightarrow \mathfrak{M}_{q}^{\mathrm{J}}[\delta]$ described in (30). Geometrically, the consistency condition means that the quantum instantons $\nabla_{\mathrm{I}}$ and $\nabla_{\mathrm{J}}$ coincide in the "intersection" variety $\mathfrak{M}_{q}^{\mathrm{I}}\left[\delta^{-1}\right] \simeq \mathfrak{M}_{q}^{\mathrm{J}}[\delta]$, up to a gauge transformation.

The goal of this Section is to convert ADHM data into a consistent pair of quantum instantons, in close analogy with the classical case.

Quantum Instantons on quantum Minkowski space-time As before, let $V, W$ denote Hermitian vector spaces of dimension $c$ and $n$, respectively. Let $\tilde{W}=V \oplus V \oplus W$.

Let $\left(B_{1}, B_{2}, i, j\right)$ be an ADHM datum, as in (48). We start by considering the following sequence of free $\mathfrak{M}_{q}^{\mathrm{I}}$-modules:

$$
V \otimes \mathfrak{M}_{q}^{\mathrm{I}} \stackrel{\alpha_{\mathrm{I}}}{\longrightarrow} \tilde{W} \otimes \mathfrak{M}_{q}^{\mathrm{I}} \stackrel{\beta_{\mathrm{I}}}{\longrightarrow} V \otimes \mathfrak{M}_{q}^{\mathrm{I}}
$$


where the maps $\alpha_{\mathrm{I}}$ and $\beta_{\mathrm{I}}$ are given by:

$$
\alpha_{\mathrm{I}}=\left(\begin{array}{c}
B_{1} \otimes \mathbf{1}-\mathbf{1} \otimes x_{21^{\prime}} \\
B_{2} \otimes \mathbf{1}-\mathbf{1} \otimes x_{22^{\prime}} \\
j \otimes \mathbf{1}
\end{array}\right)
$$

and

$$
\beta_{\mathrm{I}}=\left(-B_{2} \otimes \mathbf{1}+\mathbf{1} \otimes x_{22^{\prime}} \quad B_{1} \otimes \mathbf{1}-\mathbf{1} \otimes x_{21^{\prime}} \quad i \otimes \mathbf{1}\right)
$$

The induced dual maps $\beta_{\mathrm{I}}^{\dagger}: V \otimes \mathfrak{M}_{q}^{\mathrm{I}} \rightarrow \tilde{W} \otimes \mathfrak{M}_{q}^{\mathrm{I}}$ and $\alpha_{\mathrm{I}}^{\dagger}: \tilde{W} \otimes \mathfrak{M}_{q}^{\mathrm{I}} \rightarrow$ $V \otimes \mathfrak{M}_{q}^{\mathrm{I}}$ are given by:

$$
\beta_{\mathrm{I}}^{\dagger}=\left(\begin{array}{c}
-B_{2}^{\dagger} \otimes \mathbf{1}+\mathbf{1} \otimes x_{11^{\prime}} \\
B_{1}^{\dagger} \otimes \mathbf{1}+\mathbf{1} \otimes x_{12^{\prime}} \\
i^{\dagger} \otimes \mathbf{1}
\end{array}\right)
$$

and

$$
\alpha_{\mathrm{I}}^{\dagger}=\left(B_{1}^{\dagger} \otimes \mathbf{1}+\mathbf{1} \otimes x_{12^{\prime}} \quad B_{2}^{\dagger} \otimes \mathbf{1}-\mathbf{1} \otimes x_{11^{\prime}} \quad j^{\dagger} \otimes \mathbf{1}\right)
$$

Proposition 7. 1. $\beta_{\mathrm{I}} \alpha_{\mathrm{I}}=0$ if and only if $\left[B_{1}, B_{2}\right]+i j=0$.

2. $\beta_{\mathrm{I}} \beta_{\mathrm{I}}^{\dagger}=\alpha_{\mathrm{I}}^{\dagger} \alpha_{\mathrm{I}}$ if and only if $\left[B_{1}, B_{1}^{\dagger}\right]+\left[B_{2}, B_{2}^{\dagger}\right]+i i^{\dagger}-j^{\dagger} j=0$.

Proof. It is easy to check that:

$$
\beta_{\mathrm{I}} \alpha_{\mathrm{I}}=\left(\left[B_{1}, B_{2}\right]+i j\right) \otimes \mathbf{1}+\mathbf{1} \otimes\left[x_{22^{\prime}}, x_{21^{\prime}}\right]
$$

Since $\left[x_{22^{\prime}}, x_{21^{\prime}}\right]=0$ (see equation (21) $)$, the first statement follows.

Another straightforward calculation reveals that:

$\beta_{\mathrm{I}} \beta_{\mathrm{I}}^{\dagger}-\alpha_{\mathrm{I}}^{\dagger} \alpha_{\mathrm{I}}=\left(\left[B_{1}, B_{1}^{\dagger}\right]+\left[B_{2}, B_{2}^{\dagger}\right]+i i^{\dagger}-j^{\dagger} j\right) \otimes \mathbf{1}+\mathbf{1} \otimes\left(\left[x_{11^{\prime}}, x_{22^{\prime}}\right]-\left[x_{12^{\prime}}, x_{21^{\prime}}\right]\right)$ and the second statement follows from equation (22).

Proposition 8. 1. $\alpha_{\mathrm{I}}$ is injective.

2. $\beta_{\mathrm{I}}$ is surjective if and only if $\left(B_{1}, B_{2}, i, j\right)$ is stable. 
Proof. Let $\mathcal{X}_{1}=\mathbb{C}\left[x_{11^{\prime}}, x_{12^{\prime}}\right]$ and $\mathcal{X}_{2}=\mathbb{C}\left[x_{21^{\prime}}, x_{22^{\prime}}\right]$. Then $\mathfrak{M}_{q}^{\mathrm{I}}$ can be represented in the following way:

$$
\mathfrak{M}_{q}^{\mathrm{I}}=\mathcal{X}_{1} \otimes \mathcal{X}_{2} /(\underline{221}),(231)
$$

Clearly, $\alpha_{\mathrm{I}}$ and $\beta_{\mathrm{I}}$ can be restricted to maps

$$
V \otimes \mathcal{X}_{2} \stackrel{\alpha_{\mathrm{I}}}{\longrightarrow} \tilde{W} \otimes \mathcal{X}_{2} \stackrel{\beta_{\mathrm{I}}}{\longrightarrow} V \otimes \mathcal{X}_{2}
$$

It then follows from Nakajima (see [23], lemma 2.7) that $\left.\alpha_{\mathrm{I}}\right|_{\mathcal{X}_{2}}$ is injective and that $\left.\beta_{\mathrm{I}}\right|_{\mathcal{X}_{2}}$ is surjective if and only if $\left(B_{1}, B_{2}, i, j\right)$ is stable. Since $\alpha_{\mathrm{I}}=$ $\mathbf{1}_{\mathcal{X}_{1}} \otimes \alpha_{\mathrm{I}} \mid \mathcal{X}_{2}$ and $\beta_{\mathrm{I}}=\mathbf{1}_{\mathcal{X}_{1}} \otimes \beta_{\mathrm{I}} \mid \mathcal{X}_{2}$, we conclude that $\alpha_{\mathrm{I}}$ is also injective, and that $\beta_{\mathrm{I}}$ is surjective if and only if $\left(B_{1}, B_{2}, i, j\right)$ is stable.

Thus we have the short sequence of free $\mathfrak{M}_{q}^{\mathrm{I}}$-modules:

$$
0 \rightarrow V \otimes \mathfrak{M}_{q}^{\mathrm{I}} \stackrel{\alpha_{\mathrm{I}}}{\longrightarrow} \tilde{W} \otimes \mathfrak{M}_{q}^{\mathrm{I}} \stackrel{\beta_{\mathrm{I}}}{\longrightarrow} V \otimes \mathfrak{M}_{q}^{\mathrm{I}} \rightarrow 0
$$

which is exact on the first and last terms. Its middle cohomology $E_{\mathrm{I}}=$ $\operatorname{ker} \beta_{\mathrm{I}} / \operatorname{Im} \alpha_{\mathrm{I}}$ is then a well defined right $\mathfrak{M}_{q}^{\mathrm{I}}$-module since both $\beta_{\mathrm{I}}$ and $\alpha_{\mathrm{I}}$ are right linear. Clearly $E_{\mathrm{I}}$ is torsion-free, since it is a submodule of a free module; we argue that it is projective.

Furthermore, notice that the pairing (154) also induces a pairing $E_{\mathrm{I}} \times E_{\mathrm{I}} \rightarrow$ $\mathfrak{M}_{q}^{\mathrm{I}}$.

Proposition 9. 1. $\beta_{\mathrm{I}}^{\dagger}$ is injective.

2. $\alpha_{\mathrm{I}}^{\dagger}$ is surjective if and only if $\left(B_{1}, B_{2}, i, j\right)$ is costable.

Proof. Same argument as Proposition 8 , just replacing $\mathcal{X}_{2}$ by $\mathcal{X}_{1}$ and viceversa.

Proposition 10. $\xi_{\mathrm{I}}=\beta_{\mathrm{I}} \beta_{\mathrm{I}}^{\dagger}=\alpha_{\mathrm{I}}^{\dagger} \alpha_{\mathrm{I}}$ is an isomorphism if and only if $\left(B_{1}, B_{2}, i, j\right)$ is stable. 
Proof. Applying Proposition [6] to the map $\beta_{\mathrm{I}}^{\dagger}$, we get that:

$$
\tilde{W} \otimes \mathfrak{M}_{q}^{\mathrm{I}}=\operatorname{Im} \beta_{\mathrm{I}}^{\dagger} \oplus \operatorname{ker} \beta_{\mathrm{I}}
$$

In particular, $\operatorname{Im} \beta_{\mathrm{I}}^{\dagger} \cap \operatorname{ker} \beta_{\mathrm{I}}=\{0\}$ so that the injectivity of $\xi_{\mathrm{I}}$ follows from the injectivity of $\beta_{\mathrm{I}}^{\dagger}$.

Now if $\left(B_{1}, B_{2}, i, j\right)$ is stable then $\beta_{\mathrm{I}}$ is surjective, so given $\nu \in V \otimes \mathfrak{M}_{q}^{\mathrm{I}}$, there is $\mu \in \tilde{W} \otimes \mathfrak{M}_{q}^{\mathrm{I}}$ such that $\beta_{\mathrm{I}}(\mu)=\nu$. By the decomposition (63), we know that $\mu=\mu^{\prime}+\mu^{\prime \prime}$ for some $\mu^{\prime} \in \operatorname{Im} \beta_{\mathrm{I}}^{\dagger}$ and $\mu^{\prime \prime} \in \operatorname{ker} \beta_{\mathrm{I}}$. Let $\mu^{\prime}=\beta_{\mathrm{I}}^{\dagger}\left(\nu^{\prime}\right)$. Then $\nu=\beta_{\mathrm{I}}(\mu)=\beta_{\mathrm{I}}\left(\mu^{\prime}\right)=\beta_{\mathrm{I}}\left(\beta_{\mathrm{I}}^{\dagger}\left(\nu^{\prime}\right)\right)$, hence $\xi_{\mathrm{I}}$ is also surjective.

Conversely, if $\left(B_{1}, B_{2}, i, j\right)$ is not stable, then $\beta_{\mathrm{I}}$ is not surjective, so $\beta_{\mathrm{I}} \beta_{\mathrm{I}}^{\dagger}$ is not surjective either, and $\xi_{\mathrm{I}}$ is not an isomorphism.

Now consider the Dirac operator:

$$
\begin{aligned}
\mathcal{D}_{\mathrm{I}}: \tilde{W} \otimes \mathfrak{M}_{q}^{\mathrm{I}} & \rightarrow(V \oplus V) \otimes \mathfrak{M}_{q}^{\mathrm{I}} \\
\mathcal{D}_{\mathrm{I}} & =\left(\begin{array}{c}
\beta_{\mathrm{I}} \\
\alpha_{\mathrm{I}}^{\dagger}
\end{array}\right)
\end{aligned}
$$

Moreover, we define the Laplacian:

$$
\begin{gathered}
\Xi_{\mathrm{I}}:(V \oplus V) \otimes \mathfrak{M}_{q}^{\mathrm{I}} \rightarrow(V \oplus V) \otimes \mathfrak{M}_{q}^{\mathrm{I}} \\
\Xi_{\mathrm{I}}=\mathcal{D}_{\mathrm{I}} \mathcal{D}_{\mathrm{I}}^{\dagger}=\left(\begin{array}{cc}
\beta_{\mathrm{I}} \beta_{\mathrm{I}}^{\dagger} & 0 \\
0 & \alpha_{\mathrm{I}}^{\dagger} \alpha_{\mathrm{I}}
\end{array}\right)
\end{gathered}
$$

Proposition [10 implies that $\Xi_{\mathrm{I}}=\xi_{\mathrm{I}} \mathbf{1}_{V \oplus V}$ and $\Xi_{\mathrm{I}}$ is an isomorphism too. We can then define the projection map:

$$
\begin{gathered}
P_{\mathrm{I}}: \tilde{W} \otimes \mathfrak{M}_{q}^{\mathrm{I}} \rightarrow E_{\mathrm{I}} \\
P_{\mathrm{I}}=\mathbf{1}-\mathcal{D}_{\mathrm{I}}^{\dagger} \Xi_{\mathrm{I}}^{-1} \mathcal{D}_{\mathrm{I}}
\end{gathered}
$$

For the next two Propositions, we assume that $\left(B_{1}, B_{2}, i, j\right)$ is stable. We have:

Proposition 11. $E_{\mathrm{I}} \simeq \operatorname{ker} \mathcal{D}_{\mathrm{I}}$. In particular, $E_{\mathrm{I}}$ is a projective right $\mathfrak{M}_{q^{-}}^{\mathrm{I}}$ module. 
Proof. Given $\psi \in \operatorname{ker} \beta_{\mathrm{I}}$, we show that there is a unique $\nu \in V \otimes \mathfrak{M}_{q}^{\mathrm{I}}$ such that $\psi^{\prime}=\psi+\alpha_{\mathrm{I}}(\nu) \in \operatorname{ker} \mathcal{D}_{\mathrm{I}}$, i.e. $\beta_{\mathrm{I}}\left(\psi^{\prime}\right)=\alpha_{\mathrm{I}}^{\dagger}\left(\psi^{\prime}\right)=0$. Indeed:

$$
\begin{gathered}
\beta_{\mathrm{I}}\left(\psi^{\prime}\right)=\beta_{\mathrm{I}} \alpha_{\mathrm{I}}(\nu)=0 \\
\alpha_{\mathrm{I}}^{\dagger}\left(\psi^{\prime}\right)=0 \Leftrightarrow \alpha_{\mathrm{I}}^{\dagger}(\psi)=-\alpha_{\mathrm{I}}^{\dagger} \alpha_{\mathrm{I}}(\nu)
\end{gathered}
$$

but $\xi_{\mathrm{I}}=\alpha_{\mathrm{I}}^{\dagger} \alpha_{\mathrm{I}}$ is an isomorphism, thus $\nu=\xi_{\mathrm{I}}^{-1} \alpha_{\mathrm{I}}^{\dagger}(\psi)$, as desired.

Finally, it is easy to see that given $\nu \in \tilde{W} \otimes \mathfrak{M}_{q}^{\mathrm{I}}$, there are unique $\psi \in$ $\operatorname{ker} \mathcal{D}_{\mathrm{I}}$ and $\varphi \in \operatorname{Im} \mathcal{D}_{\mathrm{I}}^{\dagger}$ such that $\nu=\psi+\varphi$. Indeed, just take $\psi=P_{\mathrm{I}} \nu$ and $\varphi=\mathcal{D}_{\mathrm{I}}^{\dagger} \Xi_{\mathrm{I}}^{-1} \mathcal{D}_{\mathrm{I}} \nu$

In other words, we conclude that $E_{\mathrm{I}} \oplus \operatorname{Im} \mathcal{D}_{\mathrm{I}}^{\dagger}=\tilde{W} \otimes \mathfrak{M}_{q}^{\mathrm{I}}$, which implies that $E_{\mathrm{I}}$ is projective as a $\mathfrak{M}_{q}^{\mathrm{I}}$-module.

Note also that $E_{\mathrm{I}}$ is finitely generated and has rank $n=\operatorname{dim} W$.

To define the connection, let $\iota_{\mathrm{I}}: E_{\mathrm{I}} \rightarrow \tilde{W} \otimes \mathfrak{M}_{q}^{\mathrm{I}}$ denote the natural inclusion and $d: \mathfrak{M}_{q}^{\mathrm{I}} \rightarrow \Omega_{\mathfrak{M}_{q}^{I}}^{1}$ denote the quantum de Rham operator. We define $\nabla_{\mathrm{I}}$ via the composition:

$$
E_{\mathrm{I}} \stackrel{\iota_{\mathrm{I}}}{\longrightarrow} \tilde{W} \otimes \mathfrak{M}_{q}^{\mathrm{I}} \stackrel{1 \otimes d}{\longrightarrow} \tilde{W} \otimes \Omega_{\mathfrak{M}_{q}^{\mathrm{I}}}^{1} \stackrel{P_{\mathrm{I}} \otimes 1}{\longrightarrow} E_{\mathrm{I}} \otimes_{\mathfrak{M}_{q}^{\mathrm{I}}} \Omega_{\mathfrak{M}_{q}^{\mathrm{I}}}^{1}
$$

Proposition 12. $F_{\nabla_{\mathrm{I}}}$ is anti-self-dual.

Proof. Note that $F_{\nabla_{\mathrm{I}}}=\nabla_{\mathrm{I}} \nabla_{\mathrm{I}}=P_{\mathrm{I}} d P_{\mathrm{I}} d$; therefore given $e \in E_{\mathrm{I}}$ we have:

$$
\begin{aligned}
F_{\nabla_{\mathrm{I}}} e & =P_{\mathrm{I}}\left(d\left(\mathbf{1}-\mathcal{D}_{\mathrm{I}}^{\dagger} \Xi_{\mathrm{I}}^{-1} \mathcal{D}_{\mathrm{I}}\right) d e\right)=P_{\mathrm{I}}\left(d \mathcal{D}_{\mathrm{I}}^{\dagger} \Xi_{\mathrm{I}}^{-1}\left(d \mathcal{D}_{\mathrm{I}}\right) e\right)= \\
& =P_{\mathrm{I}}\left(\left(d \mathcal{D}_{\mathrm{I}}^{\dagger}\right) \Xi_{\mathrm{I}}^{-1}\left(d \mathcal{D}_{\mathrm{I}}\right) e+\mathcal{D}_{\mathrm{I}}^{\dagger} d\left(\Xi_{\mathrm{I}}^{-1}\left(d \mathcal{D}_{\mathrm{I}}\right) e\right)\right)= \\
& =P_{\mathrm{I}}\left(\left(d \mathcal{D}_{\mathrm{I}}^{\dagger}\right) \Xi_{\mathrm{I}}^{-1}\left(d \mathcal{D}_{\mathrm{I}}\right) e\right)
\end{aligned}
$$

for $P_{\mathrm{I}}\left(\mathcal{D}_{\mathrm{I}}^{\dagger} d\left(\Xi_{\mathrm{I}}^{-1}\left(d \mathcal{D}_{\mathrm{I}}\right) e\right)\right)=0$. Since $\Xi_{\mathrm{I}}^{-1}=\xi_{\mathrm{I}}^{-1} \mathbf{1}$, we conclude that $F_{\nabla_{\mathrm{I}}}$ is proportional to $d \mathcal{D}_{\mathrm{I}}^{\dagger} \wedge d \mathcal{D}_{\mathrm{I}}$, as a 2 -form. 
It is then a straightforward calculation to show that each entry of $d \mathcal{D}_{\mathrm{I}}^{\dagger} \wedge$

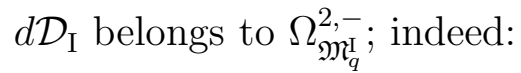

$$
\begin{gathered}
d \mathcal{D}_{\mathrm{I}}^{\dagger} \wedge d \mathcal{D}_{\mathrm{I}}=\left(\begin{array}{lr}
d x_{11^{\prime}} & -d x_{21^{\prime}} \\
d x_{12^{\prime}} & -d x_{22^{\prime}} \\
0 & 0
\end{array}\right) \wedge\left(\begin{array}{ccc}
d x_{22^{\prime}} & -d x_{21^{\prime}} & 0 \\
d x_{12^{\prime}} & -d x_{11^{\prime}} & 0
\end{array}\right)= \\
=\left(\begin{array}{ccc}
d x_{11^{\prime}} d x_{22^{\prime}}-d x_{21^{\prime}} d x_{12^{\prime}} & -d x_{11^{\prime}} d x_{21^{\prime}}+d x_{21^{\prime}} d x_{11^{\prime}} & 0 \\
d x_{12^{\prime}} d x_{22^{\prime}}-d x_{22^{\prime}} d x_{12^{\prime}} & -d x_{12^{\prime}} d x_{21^{\prime}}+d x_{22^{\prime}} d x_{11^{\prime}} & 0 \\
0 & 0 & 0
\end{array}\right)
\end{gathered}
$$

Applying the commutation relations (43), we obtain:

$$
d \mathcal{D}_{\mathrm{I}}^{\dagger} \wedge d \mathcal{D}_{\mathrm{I}}=\left(\begin{array}{ccc}
d x_{11^{\prime}} d x_{22^{\prime}}+d x_{12^{\prime}} d x_{21^{\prime}} & -2 d x_{11^{\prime}} d x_{21^{\prime}} & 0 \\
2 d x_{12^{\prime}} d x_{22^{\prime}} & -\left(d x_{11^{\prime}} d x_{22^{\prime}}+d x_{12^{\prime}} d x_{21^{\prime}}\right) & 0 \\
0 & 0 & 0
\end{array}\right)
$$

Comparing with (47), we have proved our claim.

Gauge equivalence We show that if $\left(B_{1}, B_{2}, i, j\right)$ and $\left(B_{1}^{\prime}, B_{2}^{\prime}, i^{\prime}, j^{\prime}\right)$ are equivalent $\mathrm{ADHM}$ data, then the respective pairs $\left(E_{\mathrm{I}}, \nabla_{\mathrm{I}}\right)$ and $\left(E_{\mathrm{I}}^{\prime}, \nabla_{\mathrm{I}}^{\prime}\right)$ are gauge equivalent, in the sense that there is a $\mathfrak{M}_{q}^{\mathrm{I}}$-isomorphism $G: E_{\mathrm{I}}^{\prime} \rightarrow E_{\mathrm{I}}$ such that $\nabla_{\mathrm{I}}^{\prime}=G^{-1} \nabla_{\mathrm{I}} G$.

To do that, recall that $\left(B_{1}, B_{2}, i, j\right)$ and $\left(B_{1}^{\prime}, B_{2}^{\prime}, i^{\prime}, j^{\prime}\right)$ are equivalent if there exists $g \in \mathrm{U}(V)$ such that:

$$
B_{k}^{\prime}=g B_{k} g^{-1}, k=1,2 \quad i^{\prime}=g i \quad j^{\prime}=j g^{-1}
$$

Let $G \in U(\tilde{W})$ be given by $g \times g \times \mathbf{1}_{W}$. It is then easy to check that the following diagram is commutative:

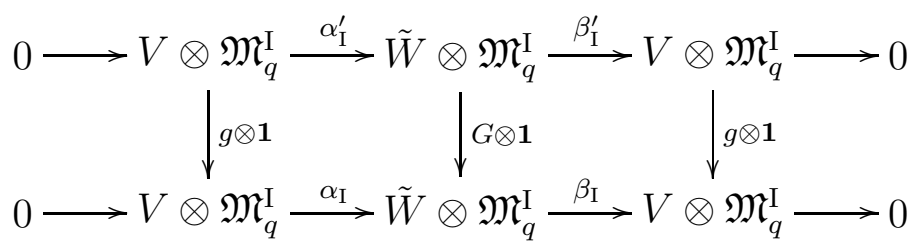


Therefore the modules $E_{\mathrm{I}}=\operatorname{ker} \beta_{\mathrm{I}} / \operatorname{Im} \alpha_{\mathrm{I}}$ and $E_{\mathrm{I}}^{\prime}=\operatorname{ker} \beta_{\mathrm{I}}^{\prime} / \operatorname{Im} \alpha_{\mathrm{I}}^{\prime}$ are isomorphic; indeed, it is easy to see that $G$ maps $E_{\mathrm{I}}^{\prime}$ onto $E_{\mathrm{I}}$ (regarded as submodules of $\left.\tilde{W} \otimes \mathfrak{M}^{\mathrm{I}}\right)$. We shall also denote by $G$ the induced isomorphism $\left.E_{\mathrm{I}}^{\prime} \rightarrow E_{\mathrm{I}}\right)$.

Now denote by $\iota_{\mathrm{I}}^{\prime}: E_{\mathrm{I}}^{\prime} \rightarrow \tilde{W} \otimes \mathfrak{M}_{q}^{\mathrm{I}}$ the inclusion and by $P_{\mathrm{I}}^{\prime}: \tilde{W} \otimes \mathfrak{M}_{q}^{\mathrm{I}} \rightarrow E_{\mathrm{I}}^{\prime}$ the projection (66). Clearly, $\iota_{\mathrm{I}}^{\prime}=G^{-1} \iota_{\mathrm{I}} G$ and $P_{\mathrm{I}}^{\prime}=G^{-1} P_{\mathrm{I}} G$. In addition, we have:

$$
\begin{aligned}
\nabla_{\mathrm{I}}^{\prime} & =P_{\mathrm{I}}^{\prime} d \iota_{\mathrm{I}}^{\prime}=G^{-1} P_{\mathrm{I}} G d G^{-1} \iota_{\mathrm{I}} G= \\
& =G^{-1} P_{\mathrm{I}}\left(G d G^{-1}\left(\iota_{\mathrm{I}} G\right)+d \iota_{\mathrm{I}} G\right)= \\
& =G^{-1} P_{\mathrm{I}} d \iota_{\mathrm{I}} G=G^{-1} \nabla_{\mathrm{I}} G
\end{aligned}
$$

since $G$ acts as the identity on $\mathfrak{M}_{q}^{\mathrm{I}}$, so that $d G^{-1}=0$.

Real structure Dualizing the monad (62) and using the identifications $\left(V \otimes \mathfrak{M}_{q}^{\mathrm{I}}\right)^{\dagger} \simeq V \otimes \mathfrak{M}_{q}^{\mathrm{I}}$ and $\left(\tilde{W} \otimes \mathfrak{M}_{q}^{\mathrm{I}}\right)^{\dagger} \simeq \tilde{W} \otimes \mathfrak{M}_{q}^{\mathrm{I}}$ one obtains:

$$
0 \rightarrow V \otimes \mathfrak{M}_{q}^{\mathrm{I}} \stackrel{\beta_{\perp}^{\dagger}}{\rightarrow} \tilde{W} \otimes \mathfrak{M}_{q}^{\mathrm{I}} \stackrel{\alpha_{\perp}^{\dagger}}{\rightarrow} V \otimes \mathfrak{M}_{q}^{\mathrm{I}} \rightarrow 0
$$

From Proposition 9, this monad is again exact at the first and last terms. Let us denote its cohomology by $E_{\mathrm{I}}^{\dagger}$, which also has the structure of a right $\mathfrak{M}_{q}^{\mathrm{I}}$-module. Moreover, via the procedure in the proof of Proposition [1], $E_{\mathrm{I}}^{\dagger}$ can be identified with the kernel of the map:

$$
\left(\begin{array}{c}
\alpha_{\mathrm{I}}^{\dagger} \\
\beta_{\mathrm{I}}
\end{array}\right): \tilde{W} \otimes \mathfrak{M}_{q}^{\mathrm{I}} \rightarrow(V \oplus V) \otimes \mathfrak{M}_{q}^{\mathrm{I}}
$$

which is clearly isomorphic to $\operatorname{ker} \mathcal{D}_{\mathrm{I}} \simeq E_{\mathrm{I}}$. Therefore, the involution $\dagger$ : $\tilde{W} \otimes \mathfrak{M}_{q}^{\mathrm{I}} \rightarrow \tilde{W} \otimes \mathfrak{M}_{q}^{\mathrm{I}}$ induces a map $\dagger: E_{\mathrm{I}} \rightarrow E_{\mathrm{I}}$; the desired property follows easily from (56). To check the compatibility of $\dagger$ with the connection $\nabla_{\mathrm{I}}$, note that:

$$
\nabla_{\mathrm{I}}\left(e^{\dagger}\right)=P_{\mathrm{I}} d\left(e^{\dagger}\right)=P_{\mathrm{I}}(d e)^{\dagger}=\left(P_{\mathrm{I}} d e\right)^{\dagger}=\left(\nabla_{\mathrm{I}} e\right)^{\dagger}
$$

Summing up the work done so far, we have constructed a well-defined map from the set of equivalence classes of classical ADHM data to the set of gauge equivalence classes of quantum instantons on quantum Minkowski space-time $\mathfrak{M}_{q}^{\mathrm{I}}$, in the sense of Definition 3.3 
Connection matrix Finally, let us describe the connection matrix associated with the connection $\nabla_{\text {I }}$ given above. To do that, let $\left\{\sigma_{k}, \rho^{k}\right\}_{k=1}^{r}$ be a dual basis for $E_{\mathrm{I}}$. Let also $\left\{w_{k}\right\}_{k=1}^{r}$ be an orthonormal basis for $W$. These choices induce a natural map:

$$
\begin{aligned}
\Psi: W \otimes \mathfrak{M}_{q}^{\mathrm{I}} & \rightarrow \tilde{W} \otimes \mathfrak{M}_{q}^{\mathrm{I}} \\
\Psi\left(w_{k} \otimes f\right) & =\iota_{\mathrm{I}}\left(\sigma_{k}\right) f
\end{aligned}
$$

extended by linearity. Then $\Psi$ has the property $\mathcal{D}_{\mathrm{I}} \circ \Psi=0$ :

$$
\mathcal{D}_{\mathrm{I}} \circ \Psi\left(\sum_{k} w_{k} \otimes f_{k}\right)=\mathcal{D}_{\mathrm{I}}\left(\sum_{k} f_{k} \iota\left(\sigma_{k}\right)\right)=\sum f_{k} \mathcal{D}_{\mathrm{I}}\left(\iota\left(\sigma_{k}\right)\right)=0
$$

The map $\Psi$ is clearly injective, so that $\Psi^{\dagger} \Psi: W \otimes \mathfrak{M}_{q}^{\mathrm{I}} \rightarrow W \otimes \mathfrak{M}_{q}^{\mathrm{I}}$ is an isomorphism. Moreover, the basis $\left\{w_{k}\right\}_{k=1}^{r}$ can be chosen such that $\Psi^{\dagger} \Psi=\mathbf{1}$.

On the other hand, consider the map:

$$
\begin{aligned}
\rho: E_{\mathrm{I}} & \rightarrow W \otimes \mathfrak{M}_{q}^{\mathrm{I}} \\
\rho(e) & =\sum_{k} w_{k} \otimes \rho^{k}(e)
\end{aligned}
$$

Then $\rho P_{\mathrm{I}} \Psi=\mathbf{1}$, thus $\rho P_{\mathrm{I}}=\Psi^{\dagger}$.

Recalling that the entries of the connection matrix are given by $A_{k}^{l}=$ $\rho^{l} \otimes \mathbf{1}\left(\nabla_{\mathrm{I}} \sigma_{k}\right)$, we have:

$$
A_{k}^{l}=\rho^{l}\left(P_{\mathrm{I}} d \Psi\left(w_{k} \otimes 1\right)\right)
$$

But the right hand side are just the matrix coefficients of $\Psi^{\dagger} d \Psi$, so that $A=\Psi^{\dagger} d \Psi$, as in the classical ADHM construction.

Quantum instantons on $S_{q}^{\mathrm{J}} \quad$ Consider the monad

$$
0 \rightarrow V \otimes \mathfrak{M}_{q}^{\mathrm{J}} \stackrel{\alpha_{\Im}}{\rightarrow} \tilde{W} \otimes \mathfrak{M}_{q}^{\mathrm{J}} \stackrel{\beta_{\mathrm{J}}}{\rightarrow} V \otimes \mathfrak{M}_{q}^{\mathrm{J}} \rightarrow 0
$$

with the maps:

$$
\alpha_{\mathrm{J}}=\left(\begin{array}{c}
B_{1} \otimes \mathbf{1}-\mathbf{1} \otimes y_{12^{\prime}} \\
B_{2} \otimes \mathbf{1}-\mathbf{1} \otimes y_{22^{\prime}} \\
j \otimes \mathbf{1}
\end{array}\right)
$$


and

$$
\beta_{\mathrm{J}}=\left(-B_{2} \otimes \mathbf{1}+\mathbf{1} \otimes y_{22^{\prime}} \quad B_{1} \otimes \mathbf{1}-\mathbf{1} \otimes y_{12^{\prime}} \quad i \otimes \mathbf{1}\right)
$$

so that $\alpha_{\mathrm{J}}=(\mathbf{1} \otimes \eta) \alpha_{\mathrm{I}}\left(\mathbf{1} \otimes \eta^{-1}\right)$ and $\beta_{\mathrm{J}}=(\mathbf{1} \otimes \eta) \beta_{\mathrm{I}}\left(\mathbf{1} \otimes \eta^{-1}\right)$

It is again easy to check that $\beta_{\mathrm{J}} \alpha_{\mathrm{J}}=0$, that $\alpha_{\mathrm{J}}$ is injective, and that $\beta_{\mathrm{J}}$ is surjective if and only if $\left(B_{1}, B_{2}, i, j\right)$ is stable. Thus, the cohomology of (68), denoted by $E_{\mathrm{J}}$, is a projective right $\mathfrak{M}_{q}^{\mathrm{J}}$-module. The connection $\nabla_{\mathrm{J}}$ and real structure $\dagger_{\mathrm{J}}: E_{\mathrm{J}} \rightarrow E_{\mathrm{J}}$ can be similarly defined, and we obtain a quantum instanton on $\mathfrak{M}_{q}^{\mathrm{J}}$.

Consistency If the modules $E_{\mathrm{I}}$ and $E_{\mathrm{J}}$ are constructed as above, the consistency map (57) arises in the following way. Consider the diagram:

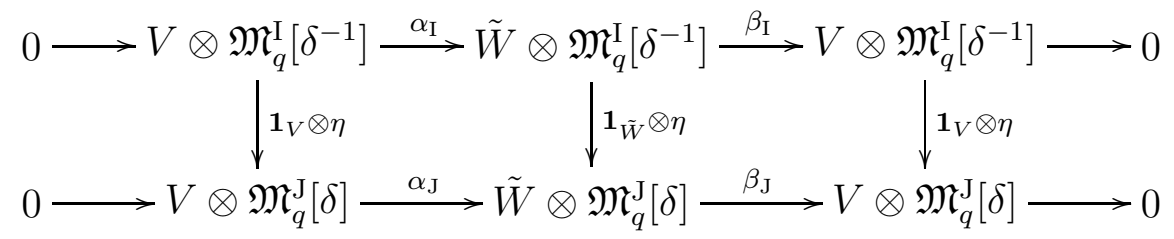

Clearly, the cohomology of the first row is $E_{\mathrm{I}}\left[\delta^{-1}\right]$, while the cohomology of the second row is $E_{\mathrm{J}}[\delta]$. Moreover, the diagram is commutative. Therefore, the isomorphism $\mathbf{1}_{\tilde{W}} \otimes \eta$ induces an isomorphism $\Gamma: E_{\mathrm{I}}\left[\delta^{-1}\right] \rightarrow E_{\mathrm{J}}[\delta]$, as required in Definition 3.3 .

To establish the consistency between the connections $\nabla_{\mathrm{I}}$ and $\nabla_{\mathrm{J}}$, it is enough to show that the connection matrices $A_{\mathrm{I}}$ and $A_{\mathrm{J}}$ are related via a gauge transformation on the "intersection algebra" $\mathfrak{M}_{q}^{\mathrm{I}}\left[\delta^{-1}\right] \stackrel{\eta}{\rightarrow} \mathfrak{M}_{q}^{\mathrm{J}}[\delta]$. Indeed, fix a trivialization: $\Psi_{\mathrm{I}}$ of $E_{\mathrm{I}}$ such that $\Psi_{\mathrm{I}}^{\dagger} \Psi_{\mathrm{I}}=1$; consider the diagram:

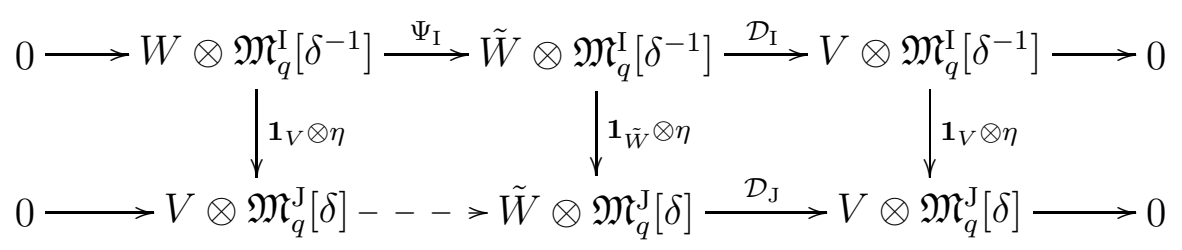

The commutativity of the second square on the above follows from the commutativity of the diagram (69). This means that $\Psi_{\mathrm{J}}=\left(\mathbf{1}_{\tilde{W}} \otimes \eta\right) \Psi_{\mathrm{I}}\left(\mathbf{1}_{V} \otimes \eta^{-1}\right)$ 
is a trivialization of $E_{\mathrm{J}}$. To simplify notation, let us simply use $\eta$ to denote $\mathbf{1} \otimes \eta$. Hence:

$$
\begin{aligned}
A_{\mathrm{J}} & =\left(\eta \Psi_{\mathrm{I}} \eta^{-1}\right)^{\dagger} d_{\mathrm{J}}\left(\eta \Psi_{\mathrm{I}} \eta^{-1}\right)=\eta \Psi_{\mathrm{I}}^{\dagger} \eta^{-1} \eta d_{\mathrm{I}}\left(\Psi_{\mathrm{I}} \eta^{-1}\right)= \\
& =\eta\left(\Psi_{\mathrm{I}}^{\dagger} d_{\mathrm{I}} \Psi_{\mathrm{I}}\right) \eta^{-1}+\eta \Psi_{\mathrm{I}}^{\dagger} \Psi_{\mathrm{I}} d_{\mathrm{I}}\left(\eta^{-1}\right)=\eta A_{\mathrm{I}} \eta^{-1}+\eta d_{\mathrm{I}} \eta^{-1}
\end{aligned}
$$

where $d_{\mathrm{I}}$ and $d_{\mathrm{J}}$ denote the de Rham operators on $\mathfrak{M}_{\mathrm{I}}$ and $\mathfrak{M}_{\mathrm{J}}$, respectively.

In other words, $\nabla_{\mathrm{J}}=\Gamma \nabla_{\mathrm{I}} \Gamma^{-1}$. We also used the fact that $\left(\eta^{-1}\right)^{\dagger}=\eta$ and that $d_{\mathrm{J}} \eta=\eta d_{\mathrm{I}}$.

Finally, recall that $\dagger \eta=\eta \dagger$. Since $\Gamma$ is induced from $\eta$ and $\dagger$ is induced from $\dagger$, we conclude that indeed: $\dagger_{J} \Gamma=\Gamma \dagger_{\mathrm{I}}$.

We sum up the work done in this Section in the following statement, which motivated the title of this paper:

Theorem 13. There exists a well-defined map from the set of equivalence classes of regular ADHM data to the moduli space of gauge equivalence classes of consistent pairs of quantum instantons.

\section{Quantum Penrose transform and further perspectives}

\subsection{Completeness conjecture and the quantum Pen- rose transform}

As we mentioned in Introduction, we conjecture that all anti-self-dual connections on $\mathfrak{M}_{q}^{\mathrm{I}}$ are gauge equivalent to the ones produced above. In other words, the map given by Theorem 13 is invertible: given a consistent pair of quantum instantons, there is an $\mathrm{ADHM}$ datum $\left(B_{1}, B_{2}, i, j\right)$ such that the consistent pair can be reconstructed from $\left(B_{1}, B_{2}, i, j\right)$ via the procedure above.

As a consequence of this conjecture, we are able to conclude that the moduli space of quantum instantons actually coincides with the moduli space of classical instantons, therefore fully justifying the title of this paper. 
The key ingredient in the proof of the classical version of this conjecture is Penrose's twistor diagram (also termed the flat self-duality diagram by Manin [19]):

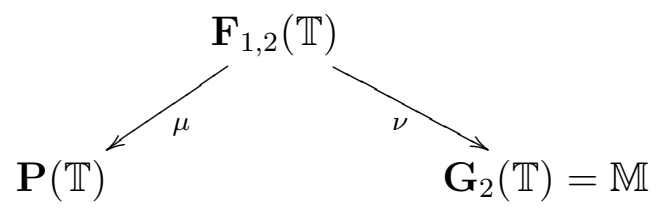

where $\mathbf{F}_{1,2}(\mathbb{T})$ denotes the flag manifold of lines within planes in $\mathbb{T}$, a 4dimensional complex vector space.

Recall also that the four sphere $S^{4}$ is naturally embedded into $\mathbb{M}$. It can be realized as the fixed point set of the following real structure $\sigma$ on $\mathbb{M}$ :

$$
\begin{array}{cc}
\sigma\left(z_{11^{\prime}}\right)=\overline{z_{22^{\prime}}} & \sigma\left(z_{12^{\prime}}\right)=\overline{z_{21^{\prime}}} \\
\sigma\left(z_{21^{\prime}}\right)=-\overline{z_{12^{\prime}}} & \sigma\left(z_{22^{\prime}}\right)=\overline{z_{11^{\prime}}} \\
\sigma(D)=\bar{D} & \sigma\left(D^{\prime}\right)=\overline{D^{\prime}}
\end{array}
$$

Moreover, we observe that even though the affine pieces $\mathbb{M}^{\mathrm{I}}$ and $\mathbb{M}^{\mathrm{J}}$ do not cover $\mathbb{M}$, all of its real points lie within their union, that is $S^{4} \hookrightarrow \mathbb{M}^{\mathrm{I}} \cup \mathbb{M}^{J}$.

We claim that there are $q$-deformations of $\mathbf{G}_{2}(\mathbb{T})$ and $\mathbf{F}_{1,2}(\mathbb{T})$ which provide a correspondence between a $q$-deformed Grassmannian and the classical twistor space $\mathbf{P}(\mathbb{T})$. Furthermore, our quantum Minkowski space-time $\mathfrak{M}_{q}^{\mathrm{I}}$ is an affine patch of the $q$-deformed Grassmannian. Moreover, all relevant noncommutative varieties can also be obtained from the quantum group $G L(4)_{q}$ extended by appropriate derivations. However, in the construction below, we will only use the quantum group $S L(2)_{q}$ enlarged by its corepresentations of functional dimension 2 and by certain degree operators.

Once these noncommutative varieties are constructed, we hope that our conjecture will be proved in complete parallel with the classical version.

Quantum Grassmannian Let us now describe the noncommutative variety from which the quantum Minkowski space-time $\mathfrak{M}_{q}^{\mathrm{I}}$ is obtained via localization. Recall that $q$ is a formal parameter. 
Definition. The quantum compactified, complexified Minkowski space $\mathfrak{M}_{p, q}$ is the associative graded $\mathbb{C}$-algebra generated by $z_{11^{\prime}}, z_{12^{\prime}}, z_{21^{\prime}}, z_{22^{\prime}}, D, D^{\prime}$ satisfying the relations (71) to 775) below $\left(p=q^{ \pm 1}\right)$ :

$$
\begin{aligned}
& z_{11^{\prime}} z_{12^{\prime}}=z_{12^{\prime}} z_{11^{\prime}} \quad z_{11^{\prime}} z_{21^{\prime}}=z_{21^{\prime}} z_{11^{\prime}} \\
& z_{12^{\prime}} z_{22^{\prime}}=z_{22^{\prime}} z_{12^{\prime}} \quad z_{21^{\prime}} z_{22^{\prime}}=z_{22^{\prime}} z_{21^{\prime}} \\
& z_{12^{\prime}} z_{21^{\prime}}=z_{21^{\prime}} z_{12^{\prime}} \\
& q^{-1}\left(z_{11^{\prime}} z_{22^{\prime}}-z_{12^{\prime}} z_{21^{\prime}}\right)=q\left(z_{22^{\prime}} z_{11^{\prime}}-z_{12^{\prime}} z_{21^{\prime}}\right) \\
& D z_{11^{\prime}}=p q^{-1} z_{11^{\prime}} D \quad D^{\prime} z_{11^{\prime}}=p^{-1} q^{-1} z_{11^{\prime}} D^{\prime} \\
& D z_{12^{\prime}}=p q^{-1} z_{12^{\prime}} D \quad D^{\prime} z_{12^{\prime}}=p^{-1} q z_{12^{\prime}} D^{\prime} \\
& D z_{21^{\prime}}=p q z_{21^{\prime}} D \quad D^{\prime} z_{21^{\prime}}=p^{-1} q^{-1} z_{21^{\prime}} D^{\prime} \\
& D z_{22^{\prime}}=p q z_{22^{\prime}} D \quad D^{\prime} z_{22^{\prime}}=p^{-1} q z_{22^{\prime}} D^{\prime} \\
& p^{-1} D D^{\prime}=p D^{\prime} D \\
& q^{-1}\left(z_{11^{\prime}} z_{22^{\prime}}-z_{12^{\prime}} z_{21^{\prime}}\right)=p^{-1} D D^{\prime}
\end{aligned}
$$

The relations (71)-(74) are simply commutation relations, while (75) plays the role of the quadric (3) that defines $\mathbb{M}$ as a subvariety of $\mathbb{P}^{5}$. In other words, the algebra $\mathfrak{M}_{p, q}$ can be regarded as a quantum Grassmannian. Note also that the relations (71)-(75) can be expressed in $R$-matrix form.

Furthermore, in analogy with the classical case, it is not difficult to establish the following:

Proposition 14. The algebras $\mathfrak{M}_{q}^{\mathrm{I}}$ and $\mathfrak{M}_{q}^{\mathrm{J}}$ are localizations of $\mathfrak{M}_{p, q}$ with respect to $D$ and $D^{\prime}$, respectively, independently on $p=q^{ \pm 1}$. In particular,

$$
x_{r s^{\prime}}=\frac{z_{r s^{\prime}}}{D} \quad \text { and } \quad y_{r s^{\prime}}=\frac{z_{r s^{\prime}}}{D^{\prime}}
$$

Proof. The proof is a straightforward calculation left to the reader. Note only that the notation $\frac{z_{r s^{\prime}}}{D}$ means $c_{r s^{\prime}}^{-1 / 2} D^{-1} z_{r s^{\prime}}=c_{r s^{\prime}}^{1 / 2} z_{r s^{\prime}} D^{-1}$ whenever $D^{-1} z_{r s^{\prime}}=$ $c_{r s^{\prime}} z_{i j^{\prime}} D^{-1}$ for some constant $c_{r s^{\prime}}$, and similarly for $\frac{z_{r s^{\prime}}}{D^{\prime}}$.

It is also important to note that it follows from the comparison of equations (73) with equations (19) and (201) that $\delta^{2}=\frac{D^{\prime}}{D}$. 
In geometric terms, our quantum Minkowski space-time $\mathfrak{M}_{q}^{\mathrm{I}}$ appears as an affine patch of the quantum Grassmannian $\mathfrak{M}_{p, q}$, thus justifying our Definition.

The quantum 4-sphere Let us define the action of the $\dagger$-involution on the generators of $\mathfrak{M}_{p, q}$ as follows:

$$
\begin{array}{ll}
z_{11^{\prime}}^{\dagger}=z_{22^{\prime}} & z_{12^{\prime}}^{\dagger}=-z_{21^{\prime}} \\
z_{21^{\prime}}^{\dagger}=-z_{12^{\prime}} & z_{22^{\prime}}^{\dagger}=z_{11^{\prime}} \\
D^{\dagger}=D & D^{\prime \dagger}=D^{\prime} \\
q^{\dagger}=q & p^{\dagger}=p
\end{array}
$$

We then extend $\dagger$ to $\mathfrak{M}_{p, q}$ by requiring it to be a conjugate linear antihomomor-phism. Note that $\dagger$ interchanges the quantum Grassmannians, i.e. $\dagger: \mathfrak{M}_{p, q} \rightarrow \mathfrak{M}_{p^{-1}, q}$. One easily checks that it is consistent with the $\dagger$-involutions previously defined on $\mathfrak{M}_{q}^{\mathrm{I}}$ and $\mathfrak{M}_{q}^{\mathrm{J}}$. Also, comparing with (70), we see that the map $\dagger$ just defined is the analogue of the real structure $\sigma$ on M. Therefore, we propose the following definition.

Definition. The quantum 4-dimensional sphere $S_{q}$ is the pair of algebras $\mathfrak{M}_{q^{ \pm 1}, q}$ equipped with the map $\dagger: \mathfrak{M}_{p, q} \rightarrow \mathfrak{M}_{p^{-1}, q}$ :

$$
S_{q}=\left(\mathfrak{M}_{q^{ \pm 1}, q}, \dagger\right)
$$

Alternative definitions of quantum 4-dimensional spheres have been proposed by several other authors, see in particular [2, 6, 7]. Note however that $\mathfrak{M}_{p, q}$ is a deformation of the algebra of homogeneous coordinates, not of the algebra of polynomial functions as in the examples discussed in the references mentioned above. Our definition is justified by the analogy with the commutative case and by the construction of quantum instantons on the two "affine patches" $S_{q}^{\mathrm{I}}$ and $S_{q}^{\mathrm{J}}$ of $S_{q}$ done in Section 3 .

Quantum flag variety Let $\mathbb{P}$ denote the projective twistor space $\mathbf{P}(\mathbb{T})$; recall that the flag variety $\mathbb{F}=\mathbf{F}_{1,2}(\mathbb{T})$ can be naturally embedded in the product $\mathbb{P} \times \mathbb{M}$ by sending the flag $[$ line $C$ plane $] \in \mathbb{F}$ to the pair (line,plane $) \in \mathbb{P} \times \mathbb{M}$. 
More precisely, let $\left[z_{k}\right]\left(k=1,2,1^{\prime}, 2^{\prime}\right.$, consistently with the splitting (2) $)$ be homogeneous coordinates in $\mathbb{P}$; then $\mathbb{F}$ can be described as an intersection of the following quadrics in $\mathbb{P} \times \mathbb{M}$ :

$$
\begin{array}{rl}
D z_{1^{\prime}}+z_{1} z_{21^{\prime}}-z_{2} z_{11^{\prime}}=0 & D z_{2^{\prime}}+z_{1} z_{22^{\prime}}-z_{2} z_{12^{\prime}}=0 \\
D^{\prime} z_{1}+z_{2^{\prime}} z_{11^{\prime}}-z_{1^{\prime}} z_{12^{\prime}}=0 & D^{\prime} z_{2}+z_{2^{\prime}} z_{21^{\prime}}-z_{1^{\prime}} z_{22^{\prime}}=0
\end{array}
$$

Let us now introduce the quantum flag variety that provides a correspondence between the quantum Grassmannian described above and the classical twistor space. Notice that the coordinate algebra of the projective twistor space $\mathbb{P}$ is simply given by:

$$
\mathfrak{P}=\mathbb{C}\left[z_{1}, z_{2}, z_{1^{\prime}}, z_{2^{\prime}}\right]_{h}
$$

where the subscript " $h$ " means that $\mathfrak{P}$ consists only of the homogeneous polynomials.

Again, the starting point lies in a natural extension of the quantum group $S U(2)_{q}$, this time by left and right corepresentations of functional dimension 2. We define left and right corepresentations of $G L(2)_{q}$ as the vector spaces $L_{q}$ and $R_{q}$ of polynomials on two noncommutative variables $g_{1}, g_{2}$ and $g_{1^{\prime}}, g_{2^{\prime}}$, respectively, satisfying the commutation relations:

$$
\begin{gathered}
g_{1} g_{2}=q^{-1} g_{2} g_{1} \\
g_{1^{\prime}} g_{2^{\prime}}=q^{-1} g_{2^{\prime}} g_{1^{\prime}}
\end{gathered}
$$

The coaction is given by, respectively:

$$
\begin{aligned}
\Delta_{L}\left(g_{r}\right) & =\sum_{k=1,2} g_{r k^{\prime}} \otimes g_{k} \\
\Delta_{R}\left(g_{s^{\prime}}\right) & =\sum_{k=1,2} g_{k^{\prime}} \otimes g_{k s^{\prime}}
\end{aligned}
$$

The commutation relations (6) and (7) for the quantum group $G L(2)_{q}$ imply that $\Delta_{L}$ and $\Delta_{R}$ are indeed corepresentations (in fact, they are also necessary conditions). 
We can also regard $L_{q}$ and $R_{q}$ as corepresentations of the quantum groups $S L(2)_{q}, \widetilde{G L}(2)_{q}$ and $\widetilde{S L}(2)_{q}$. Again, we will be primarily interested in $\widetilde{S L}(2)_{q}$.

We define the $\dagger$-involution on the generators of $L_{q}$ and $R_{q}$ as follows:

$$
\begin{aligned}
g_{1}^{\dagger} & =g_{2}, & g_{2}^{\dagger} & =-g_{1} \\
g_{1^{\prime}}^{\dagger} & =g_{2^{\prime}}, & g_{2^{\prime}}^{\dagger} & =-g_{1^{\prime}}
\end{aligned}
$$

As before, we extend $\dagger$ to a conjugate linear anti-homomorphisms of $L_{q}$ and $R_{q}$; see page 11. We also get:

$$
\Delta_{L}\left(x^{\dagger}\right)=\left(\Delta_{L}(x)\right)^{\dagger} \otimes\left(\Delta_{L}(x)\right)^{\dagger}, \quad \text { and } \quad \Delta_{R}\left(y^{\dagger}\right)=\left(\Delta_{R}(y)\right)^{\dagger} \otimes\left(\Delta_{R}(y)\right)^{\dagger}
$$

for $x \in L_{q}$ and $y \in R_{q}$, which means that $\Delta_{L}$ and $\Delta_{R}$ are well defined as corepresentations of the quantum groups $S U(2)_{q}$ and $\widetilde{S U}(2)_{q}$.

Next, we define the semi-direct product quantum groups $\left(S L(2)_{q} \ltimes L_{q}\right)_{p}$ and $\left(R_{q} \rtimes S L(2)_{q}\right)_{p}$ as the algebras with the combined generators of $S L(2)_{q}$ and $L_{q}$ and $S L(2)_{q}$ and $R_{q}$, respectively, satisfying the relations (in $R$-matrix form):

$$
p^{1 / 2} R T_{1} S_{2}=S_{2} T_{1} \quad \text { and } \quad T_{1} S_{2}^{\prime}=S_{2}^{\prime} T_{1} R p^{1 / 2}
$$

where $R$ is the $R$-matrix given in (12) and

$$
S=\left(\begin{array}{l}
g_{1} \\
g_{2}
\end{array}\right) \quad \text { and } \quad S^{\prime}=\left(\begin{array}{c}
g_{1^{\prime}} \\
g_{2^{\prime}}
\end{array}\right)
$$

are the generating matrices for $L_{q}$ and $R_{q}$, respectively, with $S_{2}=\mathbf{1} \otimes S, S_{2}^{\prime}=$ $1 \otimes S^{\prime}$. We define a comultiplication on $\left(S L(2)_{q} \ltimes L_{q}\right)_{p}$ and $\left(R_{q} \rtimes S L(2)_{q}\right)_{p}$ by the formulas (8), (801) and (81). Remark that:

Proposition 15. The multiplication and comultiplication in $\left(S L(2)_{q} \ltimes L_{q}\right)_{p}$ and $\left(R_{q} \rtimes S L(2)_{q}\right)_{p}$ are consistent, and they yield a bialgebra structure.

The bialgebras $\left(S L(2)_{q} \ltimes L_{q}\right)_{p}$ and $\left(R_{q} \rtimes S L(2)_{q}\right)_{p}$ are quantum versions of the classical groups $S L(2) \ltimes L$ and $R \rtimes S L(2)$, respectively, where $L$ and $R$ denote left and right two-dimensional representations of $S L(2)$. It is well known that these two groups are isomorphic, where the isomorphism is 
given by the identification $L \simeq R$. It turns out that this isomorphism has a quantum analogue, and the bialgebras $\left(S L(2)_{q} \ltimes L_{q}\right)_{p}$ and $\left(R_{q} \rtimes S L(2)_{q}\right)_{p}$ are also isomorphic. The quantum isomorphism $\kappa:\left(S L(2)_{q} \ltimes L_{q}\right)_{p} \rightarrow\left(R_{q} \rtimes\right.$ $\left.S L(2)_{q}\right)_{p}$ is the identity on $S L(2)_{q}$ and acts in the generators of $L_{q}$ as follows:

$$
\begin{aligned}
& \kappa\left(g_{1}\right)=p^{-3 / 4}\left(-q^{1 / 2} g_{11^{\prime}} g_{2^{\prime}}+q^{-1 / 2} g_{12^{\prime}} g_{1^{\prime}}\right) \\
& \kappa\left(g_{2}\right)=p^{-3 / 4}\left(-q^{1 / 2} g_{21^{\prime}} g_{2^{\prime}}+q^{-1 / 2} g_{22^{\prime}} g_{1^{\prime}}\right)
\end{aligned}
$$

Therefore we define $F_{p, q}$ as $R_{q} \rtimes S L(2)_{q} \ltimes L_{q}$ modulo the equivalence given by the isomorphism $\kappa$. One can compute the following commutation relations:

$$
\begin{array}{ll}
g_{1} g_{1^{\prime}}=p^{1 / 2} g_{1^{\prime}} g_{1} & g_{2} g_{1^{\prime}}=p^{1 / 2} g_{1^{\prime}} g_{2} \\
g_{1} g_{2^{\prime}}=p^{1 / 2} g_{2^{\prime}} g_{1} & g_{2} g_{2^{\prime}}=p^{1 / 2} g_{2^{\prime}} g_{2}
\end{array}
$$

We proceed by adjoining two new generators $\partial$ and $\partial^{\prime}$ to $F_{p, q}$, and postulating the following relations (keeping in mind that $p=q^{ \pm 1}$ ):

$$
\begin{array}{rlrl}
\partial g_{11^{\prime}}=q^{-1 / 2} g_{11^{\prime}} \partial & \partial^{\prime} g_{11^{\prime}} & =q^{-1 / 2} g_{11^{\prime}} \partial^{\prime} \\
\partial g_{12^{\prime}}=q^{-1 / 2} g_{12^{\prime}} \partial & \partial^{\prime} g_{12^{\prime}} & =q^{1 / 2} g_{12^{\prime}} \partial^{\prime} \\
\partial g_{21^{\prime}}=q^{1 / 2} g_{21^{\prime}} \partial & \partial^{\prime} g_{21^{\prime}} & =q^{-1 / 2} g_{21^{\prime}} \partial^{\prime} \\
\partial g_{22^{\prime}}=q^{1 / 2} g_{22^{\prime}} \partial & \partial^{\prime} g_{22^{\prime}} & =q^{1 / 2} g_{22^{\prime}} \partial^{\prime} \\
\partial g_{1}=p^{1 / 2} q^{-1 / 2} g_{1} \partial & \partial^{\prime} g_{1}=p^{1 / 2} g_{1} \partial^{\prime} \\
\partial g_{2}=p^{-1 / 2} q^{1 / 2} g_{2} \partial & \partial^{\prime} g_{2}=p^{1 / 2} g_{2} \partial^{\prime} \\
\partial g_{1^{\prime}}=p^{-1 / 2} g_{1^{\prime}} \partial & \partial^{\prime} g_{1^{\prime}}=p^{1 / 2} q^{-1 / 2} g_{1^{\prime}} \partial^{\prime} \\
\partial g_{2^{\prime}}=p^{-1 / 2} g_{2^{\prime}} \partial & \partial^{\prime} g_{2^{\prime}}=p^{1 / 2} q^{1 / 2} g_{2^{\prime}} \partial^{\prime} \\
\partial \partial^{\prime}=p^{1 / 2} \partial^{\prime} \partial
\end{array}
$$

We denote the extended algebra $F_{p, q}\left[\partial, \partial^{\prime}\right]$ by $\widetilde{F}_{p, q}$; this is the prototype of our quantum flag variety.

Furthermore, we also define the $\dagger$-involution on $\widetilde{F}_{p, q}$ by extending the †-involutions on $\widetilde{S L}(2)_{q}, L_{q}$ and $R_{q}$ defined on (17) and (82) and declaring:

$$
\partial^{\dagger}=\partial \quad \text { and } \quad \partial^{\prime \dagger}=\partial^{\prime}
$$


As in the construction of the quantum Minkowski space-time, we introduce a new set of variables $z_{r s^{\prime}}, z_{k}, z_{k^{\prime}}$ in the bialgebra $\widetilde{F}_{p, q}=F_{p, q}\left[\partial, \partial^{\prime}\right]$, related to the original variables $g_{r s^{\prime}}, g_{k}, g_{k^{\prime}}$ via:

$$
\frac{z_{r s^{\prime}}}{\Delta}=g_{r s^{\prime}} \quad \frac{z_{k}}{\partial}=g_{k} \quad \frac{z_{k^{\prime}}}{\partial^{\prime}}=g_{k^{\prime}}
$$

where $\Delta=p^{1 / 4} \partial^{\prime} \partial=p^{-1 / 4} \partial \partial^{\prime}$. The fractions above have the same meaning as in Proposition 14. We also set:

$$
D=\partial^{2} \quad D^{\prime}=\partial^{\prime 2}
$$

Using relations (85) and (87), one re-obtains the relations (71) [75), which define $\mathfrak{M}_{p, q}$. In other words, the quantum compactified, complexified Minkowski space $\mathfrak{M}_{p, q}$ can be regarded as a subalgebra of the extended quantum group $S L(2)_{q}\left[\partial, \partial^{\prime}\right]$. Notice in particular that the quantum quadric (75) is just the relation $\operatorname{det}_{q}(T)=1$ expressed in the new variables $z_{r s^{\prime}}$.

Now using relations (86) and (87), it is easy to check that:

$$
z_{1}, z_{2}, z_{1^{\prime}} z_{2^{\prime}} \text { commute with one another }
$$

Furthermore, we also obtain:

$$
\begin{array}{lrl}
z_{1} z_{11^{\prime}}=z_{11^{\prime}} z_{1} & z_{1} z_{12^{\prime}}=z_{12^{\prime}} z_{1} \\
z_{2} z_{21^{\prime}}=z_{21^{\prime}} z_{2} & z_{2} z_{22^{\prime}}=z_{22^{\prime}} z_{2} \\
z_{1^{\prime}} z_{11^{\prime}}=z_{11^{\prime}} z_{1^{\prime}} & z_{1^{\prime}} z_{21^{\prime}}=z_{21^{\prime}} z_{1^{\prime}} \\
z_{2^{\prime}} z_{12^{\prime}}=z_{12^{\prime}} z_{2^{\prime}} & z_{2^{\prime}} z_{22^{\prime}}=z_{22^{\prime}} z_{2^{\prime}}
\end{array}
$$

and, keeping in mind that $p=q^{ \pm 1}$ :

$$
\begin{gathered}
z_{1} z_{21^{\prime}}=p q z_{21^{\prime}} z_{1}+(1-p q) z_{11^{\prime}} z_{2} \\
z_{1} z_{22^{\prime}}=p q z_{22^{\prime}} z_{1}+(1-p q) z_{12^{\prime}} z_{2} \\
z_{2} z_{11^{\prime}}=p q^{-1} z_{11^{\prime}} z_{2}+\left(1-p q^{-1}\right) z_{21^{\prime}} z_{1} \\
z_{2} z_{12^{\prime}}=p q^{-1} z_{22^{\prime}} z_{2}+\left(1-p q^{-1}\right) z_{22^{\prime}} z_{1} \\
z_{1^{\prime}} z_{12^{\prime}}=p^{-1} q z_{12^{\prime}} z_{1^{\prime}}+\left(1-p^{-1} q\right) z_{11^{\prime}} z_{2^{\prime}} \\
z_{1^{\prime}} z_{12^{\prime}}=p^{-1} q z_{22^{\prime}} z_{1^{\prime}}+\left(1-p^{-1} q\right) z_{21^{\prime}} z_{2^{\prime}} \\
z_{2^{\prime}} z_{11^{\prime}}=p^{-1} q^{-1} z_{11^{\prime}} z_{2^{\prime}}+\left(1-p^{-1} q^{-1}\right) z_{12^{\prime}} z_{1^{\prime}} \\
z_{2^{\prime}} z_{21^{\prime}}=p^{-1} q^{-1} z_{21^{\prime}} z_{2^{\prime}}+\left(1-p^{-1} q^{-1}\right) z_{22^{\prime}} z_{1^{\prime}}
\end{gathered}
$$


The commutation relations between the degree operators $D$ and $D^{\prime}$ and the commuting generators $z_{1}, z_{2}, z_{1^{\prime}}, z_{2^{\prime}}$ are given by:

$$
\begin{array}{ll}
D z_{1}=p^{-1} q^{-1} z_{1} D & D^{\prime} z_{1}=z_{1} D^{\prime} \\
D z_{2}=p^{-1} q z_{2} D & D^{\prime} z_{2}=z_{2} D^{\prime} \\
D z_{1^{\prime}}=z_{1^{\prime}} D & D^{\prime} z_{1^{\prime}}=p q^{-1} z_{1^{\prime}} D^{\prime} \\
D z_{2^{\prime}}=z_{2^{\prime}} D & D^{\prime} z_{2^{\prime}}=p q z_{2^{\prime}} D^{\prime}
\end{array}
$$

The last set of relations below follows from the identifications induced by the isomorphism $\kappa$ and its inverse, and yields the quantum analogues of the quadrics (76) and (77) defining $\mathbb{F}$ as a subvariety of $\mathbb{P} \times \mathbb{M}$ :

$$
\begin{gathered}
D z_{1^{\prime}}=p\left(z_{11^{\prime}} z_{2}-z_{21^{\prime}} z_{1}\right) \\
D z_{2^{\prime}}=p\left(z_{12^{\prime}} z_{2}-z_{22^{\prime}} z_{1}\right) \\
D^{\prime} z_{1}=p^{-1}\left(-z_{11^{\prime}} z_{2^{\prime}}+z_{12^{\prime}} z_{1^{\prime}}\right) \\
D^{\prime} z_{2}=p^{-1}\left(-z_{21^{\prime}} z_{2^{\prime}}+z_{22^{\prime}} z_{1^{\prime}}\right)
\end{gathered}
$$

This observation motivates our next definition:

Definition. The quantum flag variety $\mathfrak{F}_{p, q}$ is the associative graded $\mathbb{C}$-algebra with generators $z_{11^{\prime}}, z_{12^{\prime}}, z_{21^{\prime}}, z_{22^{\prime}}, D, D^{\prime}, z_{1}, z_{2}, z_{1^{\prime}} z_{2^{\prime}}$ satisfying relations [71)(75) and (90)-(93) above.

In particular, note that $\mathfrak{F}_{p, q}$ can be regarded as a subalgebra of $\widetilde{F}_{p, q}$. We also remark that:

$$
\mathfrak{F}_{p, q}=\mathfrak{M}_{p, q} \otimes_{\mathbb{C}} \mathfrak{P} /(90)-(93)
$$

in close analogy with the classical case. All the relations (90, 93) can also be expressed in $R$-matrix form.

Now let

$$
\begin{aligned}
\mathfrak{F}_{q}^{\mathrm{I}} & =\mathfrak{F}_{p, q}\left[D^{-1}\right]_{0} \simeq \mathfrak{M}_{q}^{\mathrm{I}} \otimes \mathbb{C}\left[z_{1}, z_{2}\right]_{h} \\
\mathfrak{F}_{q}^{\mathrm{J}} & =\mathfrak{F}_{p, q}\left[D^{\prime-1}\right]_{0} \simeq \mathfrak{M}_{q}^{\mathrm{J}} \otimes \mathbb{C}\left[z_{1}, z_{2}\right]_{h}
\end{aligned}
$$

where $\mathfrak{F}_{p, q}\left[D^{-1}\right]$ and $\mathfrak{F}_{p, q}\left[D^{\prime-1}\right]$ denote the localization of $\mathfrak{F}_{p, q}$ as a $\mathfrak{M}_{p, q^{-}}$ bimodule and the subscript "0" means that we take only the degree zero part of the localized graded algebra, and the subscript " $h$ " means that only the 
homogeneous polynomials are considered. Geometrically, notice that these algebras are playing the roles of $q$-deformations of the "affine" flag varieties $\mathbb{F}^{\mathrm{I}}=\nu^{-1}\left(\mathbb{M}^{\mathrm{I}}\right)=\mathbb{M}^{\mathrm{I}} \times \mathbb{P}^{1}$ and $\mathbb{F}^{\mathrm{J}}=\nu^{-1}\left(\mathbb{M}^{\mathrm{J}}\right)=\mathbb{M}^{\mathrm{J}} \times \mathbb{P}^{1}$ [31]. To further justify Definition 4.1 we prove:

Theorem 16. The maps $\mathrm{m}_{p, q}: \mathfrak{P} \rightarrow \mathfrak{F}_{p, q}$ and $\mathrm{n}_{p, q}: \mathfrak{M}_{p, q} \rightarrow \mathfrak{F}_{p, q}$ defined as identities on the generators are injective homomorphisms. Furthermore, the multiplication in the associative algebras $\mathfrak{M}_{p, q}, \mathfrak{M}_{p, q}^{\mathrm{I}}, \mathfrak{M}_{p, q}^{\mathrm{J}}$ and $\mathfrak{F}_{p, q}, \mathfrak{F}_{p, q}^{\mathrm{I}}, \mathfrak{F}_{p, q}^{\mathrm{J}}$ described above is consistent, and they have the same Hilbert polynomials as their commutative counterparts $\mathbb{M}, \mathbb{M}^{\mathrm{I}}, \mathbb{M}^{\mathrm{J}}$ and $\mathbb{F}, \mathbb{F}^{\mathrm{I}}, \mathbb{F}^{\mathrm{J}}$, respectively.

Proof. The statement regarding the maps $\mathrm{m}_{p, q}$ and $\mathrm{n}_{p, q}$ is clear from our construction, see (94).

For the second part of the Theorem, we must first check the consistency of multiplication in the algebra $\mathfrak{M}_{p, q}$ by embedding it into its localization $\mathfrak{M}_{p, q}\left[D^{-1}\right]$. The commutation relations (71), (172) and the first column of (73) allow us to choose a basis in $\mathbb{C}\left[z_{r s^{\prime}}, D^{ \pm 1}\right]$ of the form:

$$
z_{11^{\prime}}^{n_{11^{\prime}}} z_{12^{\prime}}^{n_{12^{\prime}}} z_{21^{\prime}}^{n_{21^{\prime}}} z_{22^{\prime}}^{n_{22^{\prime}}} D^{n}, \quad \text { with } \quad n_{r s^{\prime}} \in \mathbb{Z}_{+}, \quad n \in \mathbb{Z}
$$

Inverting $D$ in (75) we obtain an expression for $D^{\prime}$, and we can check directly the second column of (73) and relation (174). Thus the basis (95) is in fact a basis of $\mathfrak{M}_{p, q}\left[D^{-1}\right]$, and its Hilbert polynomial coincides with the classical one.

Next we find the Hilbert polynomial of the algebra $\mathfrak{M}_{p, q}$. Using (75), we can present an arbitrary element from $\mathfrak{M}_{p, q}$ uniquely in the following form:

$$
P_{0}\left(z_{r s^{\prime}}\right)+P_{1}\left(z_{r s^{\prime}}, D\right) D+P_{2}\left(z_{r s^{\prime}}, D^{\prime}\right) D^{\prime}
$$

where $P_{0}, P_{1}, P_{2}$ are polynomials and $z_{r s^{\prime}}$ are ordered as in (95). This presentation coincides with the classical one, and allows to compute explicitly the Hilbert polynomial of $\mathfrak{M}_{p, q}$.

Similarly, we check the consistency of multiplication in the algebra $\mathfrak{F}_{p, q}$ by embedding it into $\mathfrak{F}_{p, q}\left[D^{-1}\right]$. In addition to the relations used above, we 
use the the first half of the commutation relations (90), (91) and the first column of (92) to choose a basis in $\mathbb{C}\left[z_{r s^{\prime}}, z_{k}, D^{ \pm 1}\right]$ of the form:

$$
z_{11^{\prime}}^{n_{11^{\prime}}} z_{12^{\prime}}^{n_{12^{\prime}}} z_{21^{\prime}}^{n_{21^{\prime}}} z_{22^{\prime}}^{n_{22^{\prime}}} z_{1}^{n_{1}} z_{2}^{n_{2}} D^{n}, \quad \text { with } \quad n_{r s^{\prime}}, n_{k} \in \mathbb{Z}_{+}, \quad n \in \mathbb{Z}
$$

Inverting $D$ in (75) and also in the first half of (93), we obtain expressions for $D^{\prime}, z_{1^{\prime}}$ and $z_{2^{\prime}}$ and check directly all the other relations involving these three generators, namely the second half of the commutation relations (90), (91) and the second column of (92). We conclude that the multiplication in $\mathfrak{F}_{p, q}\left[D^{-1}\right]$ is consistent and its basis is given by (97).

Finally, we obtain the Hilbert polynomial of $\mathfrak{F}_{p, q}$ by noticing that using (75) and (93) we can present an arbitrary element of $\mathfrak{F}_{p, q}$ uniquely in the form

$$
P_{0}\left(z_{r s^{\prime}}, z_{k}, z_{k^{\prime}}\right)+P_{1}\left(z_{r s^{\prime}}, z_{k}, D\right) D+P_{2}\left(z_{r s^{\prime}}, z_{k^{\prime}}, D^{\prime}\right) D^{\prime}
$$

where $P_{0}, P_{1}, P_{2}$ are polynomials and $z_{r s^{\prime}}, z_{k}, z_{k^{\prime}}$ are ordered as in (97). Again this presentation coincides with the classical one, and yields an explicit formula for the Hilbert polynomial of $\mathfrak{F}_{p, q}$.

Alternatively, one could also check the consistency of multiplication more efficiently using the $R$-matrix formulation.

Summing up, we have constructed noncommutative varieties $\mathfrak{M}_{p, q}$ and $\mathfrak{F}_{p, q}$, thought as quantum deformations of $\mathbb{M}$ and $\mathbb{F}$, and injective morphisms $\mathrm{m}_{p, q}$ and $\mathrm{n}_{p, q}$ fitting into the following diagram:

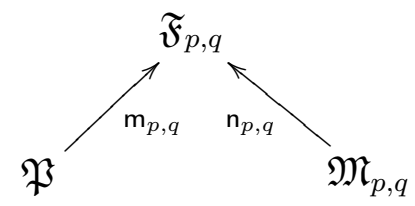

where $\mathfrak{P}$ is just the commutative projective 3-space. In analogy with the classical case, diagram (99) will be called the quantum twistor diagram. 
Furthermore, we also have the quantum local twistor diagrams:

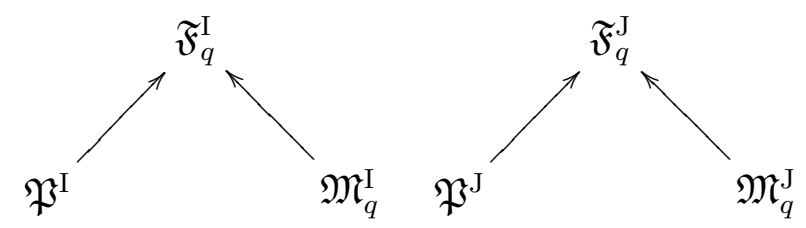

where

$$
\begin{aligned}
\mathfrak{P}^{\mathrm{I}} & =\mathbb{C}\left[z_{1}, z_{2}, x_{11^{\prime}} z_{2}-x_{21^{\prime}} z_{1}, x_{12^{\prime}} z_{2}-x_{22^{\prime}} z_{1}\right]_{h} \\
\mathfrak{P}^{\mathrm{J}} & =\mathbb{C}\left[y_{12^{\prime}} z_{1^{\prime}}-y_{11^{\prime}} z_{2^{\prime}}, y_{22^{\prime}} z_{1^{\prime}}-y_{21^{\prime}} z_{2^{\prime}}, z_{1^{\prime}}, z_{2^{\prime}}\right]_{h}
\end{aligned}
$$

as commutative subalgebras of $\mathfrak{F}_{q}^{\mathrm{I}}$ and $\mathfrak{F}_{q}^{\mathrm{J}}$, respectively. Notice that $\mathfrak{P}^{\mathrm{I}}$ and $\mathfrak{P}^{\mathrm{J}}$ are exactly the coordinate algebras of $\mu\left(\nu^{-1}\left(\mathbb{M}^{\mathrm{I}}\right)\right)$ and $\mu\left(\nu^{-1}\left(\mathbb{M}^{\mathrm{J}}\right)\right)$, the "affine" twistor spaces [31].

It is also clear from our construction that the quantum twistor diagram (99) comes with a real structure defined by the $\dagger$ involutions on $\mathfrak{F}_{p, q}$ and $\mathfrak{M}_{p, q}$ defined above, acting on the generators of $\mathfrak{P}$ as follows:

$$
z_{1}^{\dagger}=z_{2} \quad z_{2}^{\dagger}=-z_{1} \quad z_{1^{\prime}}^{\dagger}=z_{2^{\prime}} \quad z_{2^{\prime}}^{\dagger}=-z_{1^{\prime}}
$$

After rewriting the classical Penrose transform in algebraic terms, we can use the quantum twistor diagrams (99) and (100) to transform quantum objects (solutions of the quantum ASDYM equation) into classical ones (holomorphic bundles with a real structure). This is the strategy for the proof of our completeness conjecture, and a topic for a future paper.

\subsection{Remarks on roots of unity and representation the- ory}

In our construction of quantum instantons, we have specialized the formal parameter $q$ to a positive real number. Thus it is natural to ask what happens if we consider other specializations. Since our construction is deeply related with the behaviour of the Haar functional on the quantum group $S L(2)_{q}$, we expect that our results remain valid for all specializations of $q$ for which the 
Haar functional has a similar structure, namely for any complex $q$ that is not a root of unity.

It is an interesting problem to obtain a modified ADHM data for any given root of unity, and then describe the corresponding moduli space of quantum instantons in terms of this data. We expect that such description will be given by certain quiver varieties.

Moreover, equivariant anti-self-dual connections on $\mathbb{R}^{4}$ has long been a topic of intense research. In particular, instantons on the quotient spaces $\mathbb{C}^{2} / \Gamma$, where $\Gamma$ is a finite subgroup of $S U(2)$, and on their desingularizations (known as ALE spaces) have been studied by Kronheimer and Nakajima in [15, 25]. The moduli spaces of instantons on ALE spaces play a fundamental role on Nakajima's construction of representations of affine Lie algebras [24.

To obtain a quantum analogue of this construction, we first need to describe "finite subgroups" of $S U(2)_{q}$. This turns out to be a deep problem, recently solved by Ostrik [27], though only at the level of representation categories. The resulting classification, as in the classical case, is given by Dynkin diagrams of ADE type. This suggests that the corresponding moduli spaces of quantum instantons might shed a new light at Nakajima's quiver varieties and his results on representation theory.

We would also like to notice the similarities between the deformation of the representation theory for $S U(2)$ (as well as other compact simple Lie groups) and the deformation of instanton theory presented in this paper. As it is well known, the classification of irreducible corepresentations of the quantum group $S U(2)_{q}$, the behaviour of the Haar functional, the decomposition of their tensor products, etc, is the same as in the classical, non-deformed case if $q$ is not a root of unity, see [4].

The anti-self-duality condition can be viewed as half of the relations necessary to determine a representation of the quantum group $S U(2)_{q}$, and we also obtain a classification identical to the non-deformed case. Tensor products of representations of quantum groups correspond in our context to the 
composition of instantons of different ranks, and again we expect it not to depend on the quantization parameter, if it is not a root of unity.

\subsection{Further perspectives}

It is well known that the Penrose transform can be used to obtain solutions not only of the ASDYM equation, but also of a variety of other differential equations on Minkowski space-time [31]. One can then expect to define the quantum analogue of such equations in a way that our proposed quantum Penrose transform still yields the same result for generic $q$.

The most basic class of examples are the massless free field equations, which includes the wave, Dirac and Maxwell equations. In particular, the polynomial solutions to the wave equation on $\mathbb{M}^{\mathrm{I}}$ are given by the matrix coefficients of irreducible representations of $S U(2)$. Thus the appropriate quantum wave equation should have solutions on $\mathfrak{M}_{q}^{\mathrm{I}}$ given by the matrix coefficients of representations of the quantum group $S U(2)_{q}$, and a similar relation should also be true for the higher spin equations.

Moreover, the wave equation for the anti-self-dual connection plays a prominent role in the proof of completeness of instantons [19] and its solutions correspond, via Penrose transform, to certain sheaf cohomology classes defined over $\mathbf{P}(\mathbb{T})$. We expect that all this structure will be preserved under our quantum deformation of the twistor diagram, leading to a proof of our completeness conjecture.

Finally, we cannot avoid to mention another remarkable noncommutative deformation of instantons discovered by Nekrasov and Schwarz [26]. The moduli spaces of such noncommutative instantons can be regarded as a smooth compactification of the classical ADHM data, though the relation with the latter is not as straightforward as in our case [22].

A version of the Penrose transform for the Nekrasov-Schwarz noncommutative instantons has been discussed in [12. Also, it has been observed in [17] that the factorization of the noncommutative Minkowski space by a fi- 
nite subgroup $\Gamma \hookrightarrow S U(2)$ in the Nekrasov-Schwarz setting yields the smooth varieties previously introduced by Nakajima [24].

It is an interesting and challenging problem to obtain a natural compactification of the moduli spaces of quantum instantons by considering a more general type of $\mathfrak{M}_{q}^{\mathrm{I}}$-modules than those considered here, and then compare with the smooth compactification appearing in the Nekrasov-Schwarz approach. This might lead to a further extension of our understanding of the mathematical structure of space-time.

Acknowledgement. We thank Yu. Berest for a discussion. We also thank the referee for his comments on the first version of this paper.

I.F. is supported by the NSF grant DMS-0070551, and would like to thank the MSRI for its hospitality at the later stages of this project. M.J. thanks the Departments of Mathematics at Yale University and at the University of Pennsylvania.

\section{References}

[1] Atiyah, M., Drinfeld, V., Hitchin, N., Manin, Yu.: Construction of instantons. Phys. Lett. 65A, 185-187 (1978)

[2] Bonechi, F., Ciccoli, N., Tarlini, M.: Noncommutative instantons on the 4-sphere from quantum groups. Commun. Math. Phys. 226, 419432 (2002)

[3] Carow-Watamura, U., Schlieker, M., Scholl, M., Watamura, S.: Tensor representation of the quantum group $\mathrm{SL}_{q}(2, C)$ and quantum Minkowski space. Z. Phys. C48, 159-165 (1990)

[4] Chari, V., Pressley, A.: A guide to quantum groups. Cambridge: Cambridge University Press, 1994 
[5] Connes, A.: Noncommutative geometry. San Diego: Academic Press, 1994

[6] Connes, A., Landi, G.: Noncommutative manifolds, the instanton algebra and isospectral deformations. Commun. Math. Phys. 221, 141-159 (2001)

[7] Dabrowski, L., Landi, G., Masuda, T.: Instantons on the quantum 4spheres $S_{q}^{4}$. Commun. Math. Phys. 221, 161-168 (2001)

[8] Donaldson, S.: Instantons and Geometric Invariant Theory. Commun. Math. Phys. 93, 453-460 (1984)

[9] Donaldson, S., Kronheimer, P.: The geometry of four-manifolds. Oxford: Claredon Press, 1990

[10] Drinfeld, V.: Hopf algebras and the quantum Yang-Baxter equation. Soviet Math. Dokl. 32, 254-258 (1985)

[11] Faddeev, L., Reshetikhin, N., Takhtajan, L.: Quantization of Lie groups and Lie algebras. Leningrad Math. J. 1, 193-225 (1990)

[12] Kapustin, A., Kuznetsov, A., Orlov, D.: Noncommutative instantons and twistor transform. Commun. Math. Phys. 198, 689-703 (2001)

[13] Jimbo, M.: A q-difference analogue of $U(\mathfrak{G})$ and the Yang-Baxter equation. Lett. Math. Phys. 122, 63-69 (1985)

[14] Koornwinder, T.: Representations of the twisted SU(2) quantum group and some $q$-hypergeometric orthogonal polynomials. Nederl. Akad. Wetensch. Indag. Math. 51, 97-117 (1989)

[15] Kronheimer, P., Nakajima, H.: Yang-Mills instantons and ALE gravitational instantons. Math. Ann. 288, 263-307 (1990) 
[16] Lam, T.: A first course in noncommutative rings. Second edition. New York: Springer-Verlag, 2001

[17] Lazaroiu, C.: A non-commutative geometric interpretation of the resolution of equivariant instanton moduli spaces. Preprint hep-th/9805132

[18] Majid, S.: Examples of braided groups and braided matrices. J. Math. Phys 32, 3246-3253 (1991)

[19] Manin, Yu.: Gauge field theory and complex geometry. Berlin: SpringerVerlag, 1988

[20] Masuda, T., Mimachi, K., Nakagami, Y., Noumi, M., Ueno, K.: Representations of the quantum group $\mathrm{SU}_{q}(2)$ and the little $q$-Jacobi polynomials. J. Funct. Anal. 99, 357-386 (1991)

[21] Minkowski, H.: Raum und Zeit. Phys. Z. 10, 104 (1909)

[22] Nakajima, H.: Resolutions of moduli spaces of ideal instantons on $\mathbb{R}^{4}$. In Fukaya, K., Furuta, M., Kohno, T., Kotschick, D. (eds.): Topology, geometry and field theory, pp. 129-136. River Edge, NJ: World Sci. Publishing, 1994

[23] Nakajima, H.: Lectures on Hilbert schemes of points on surfaces. Providence: American Mathematical Society, 1999

[24] Nakajima, H.: Instantons on ALE spaces, quiver varieties, and KacMoody algebras. Duke Math. J. 76, 365-416 (1994)

[25] Nakajima, H.: Moduli spaces of anti-self-dual connections on ALE gravitational instantons. Invent. Math. 102, 267-303 (1990)

[26] Nekrasov, N., Schwarz, A.: Instantons on noncommutative $R^{4}$ and $(2,0)$ superconformal six dimensional theory. Commun. Math. Phys. 221, 385432 (2001) 
[27] Ostrik, V.: Module categories, weak Hopf algebras and modular invariants. Preprint math.QA/0111139

[28] Penrose, R.: The twistor programme. Rep. Math. Phys. 12, 65-76 (1977)

[29] Schmidke, W., Wess, J., Zumino, B.: q-deformed Lorentz algebra. Zeit. Phys. C48, 471-476 (1991)

[30] Sudbery, A.: Canonical differential calculus on quantum general linear groups and supergroups. Phys. Lett. 284B, 61-65 (1992)

[31] Ward, R., Wells, R.: Twistor geometry and field theory. Cambridge: Cambridge University Press (1990)

[32] Wess, J., Zumino, B.: Covariant differential calculus on the quantum hyperplane. Nucl. Phys. B. Proc. Suppl. 18B, 302-312 (1990)

[33] Woronowicz, S.: Compact matrix pseudogroups. Commun. Math. Phys. 111, 613-665 (1987)

[34] Woronowicz, S.: Differential calculus on compact matrix pseudogroups (quantum groups). Commun. Math. Phys. 122, 125-170 (1989)

[35] Zupnik, B.: Solution of self-duality equation in quantum-group gauge theory. JETP Lett. 61, 443-447 (1995) 